\title{
Lung Involvements in Rheumatic Diseases: Update on the Epidemiology, Pathogenesis, Clinical Features, and Treatment
}

\author{
You-Jung Ha, Yun Jong Lee, and Eun Ha Kang ${ }^{D}$ \\ Division of Rheumatology, Department of Internal Medicine, Seoul National University Bundang Hospital, \\ Seongnam, Republic of Korea \\ Correspondence should be addressed to Eun Ha Kang; kangeh@snubh.org
}

Received 29 November 2017; Accepted 27 March 2018; Published 8 May 2018

Academic Editor: Paolo Spagnolo

Copyright (C) 2018 You-Jung Ha et al. This is an open access article distributed under the Creative Commons Attribution License, which permits unrestricted use, distribution, and reproduction in any medium, provided the original work is properly cited.

Lung illness encountered in patients with rheumatic diseases bears clinical significance in terms of increased morbidity and mortality as well as potential challenges placed on patient care. Although our understanding of natural history of this important illness is still limited, epidemiologic knowledge has been accumulated during the past decade to provide useful information on the risk factors and prognosis of lung involvements in rheumatic diseases. Moreover, the pathogenesis particularly in the context of genetics has been greatly updated for both the underlying rheumatic disease and associated lung involvement. This review will focus on the current update on the epidemiologic and genetics features and treatment options of the lung involvements associated with four major rheumatic diseases (rheumatoid arthritis, systemic sclerosis, myositis, and systemic lupus erythematosus), with more attention to a specific form of involvement or interstitial lung disease.

\section{Introduction}

The lung is a frequent target of autoimmune mediated injury in patients with rheumatic diseases. Rheumatic disease associated lung involvement is a major determinant of morbidity and mortality in these patients. It shows a considerable heterogeneity in incidence and prevalence, severity, and the components of the involved lung structure depending on the underlying rheumatic disease and each rheumatic disease is associated with a characteristic pattern of their lung disease (Table 1) [1]. For example, up to 70 90\% of patients with systemic sclerosis (SSc) or myositis exhibit lung involvement in the form of interstitial lung disease (ILD) while, in rheumatoid arthritis (RA) and systemic lupus erythematosus (SLE), the spectrum of pulmonary manifestations is quite broad involving almost every component of the lung structure or upper airway tracts. Furthermore, the clinical manifestation and severity of lung disease vary from subclinical abnormality to respiratory failure and death even within patients suffering the same rheumatic disease. The pulmonary manifestation could be the first clue to predict future or diagnose underlying rheumatic disease or it could occur later during the disease course. Although autoimmune mediated lung injury is thought to be a common mechanism, the key immune cells and cytokines driving the lung disease could be different depending on the underlying rheumatic disease.

Among many diverse forms of rheumatic disease associated lung involvements, most common is ILD. The histopathologic classification of rheumatic disease associated ILD (RD-ILD) follows 2013 revised American Thoracic Society/European Respiratory Society classification of idiopathic interstitial pneumonia, including usual interstitial pneumonia (UIP), nonspecific interstitial pneumonia (NSIP), cryptogenic organizing pneumonia (COP), diffuse alveolar hemorrhage (DAH), and others [2]. The histologic hallmark of UIP is a temporal heterogeneity with alternating areas of normal lung, interstitial inflammation, fibroblast foci, and honeycomb changes, which correspond to high resolution computed tomography (HRCT) findings of peripheral basilar predominant reticular abnormalities, honeycombing, traction bronchiectasis, and minimal to no ground glass opacities (GGOs) [3]. On the other hand, NSIP shows a relatively uniform appearance at low magnification due to a cellular interstitial infiltrate of mononuclear inflammatory cells 
TABLE 1: Spectrum and relative prevalence of lung involvements in rheumatic diseases.

\begin{tabular}{lccccc}
\hline & \multicolumn{2}{c}{ Parenchymal } & Pleural & \multicolumn{2}{c}{ Vascular } \\
& ILD & Airways & ++ & PAH & + \\
\hline Rheumatoid arthritis & ++ & ++ & - & - & + \\
Systemic sclerosis & +++ & - & - & ++ & + \\
Myositis & +++ & - & ++ & + & + \\
Systemic lupus erythematosus & + & + & ++ \\
\hline
\end{tabular}

The signs show relative prevalence of each manifestation (none: -, low: +, medium: ++, and high: +++); ILD: interstitial lung disease; DAH: diffuse alveolar hemorrhage; PAH: pulmonary arterial hypertension (cited and modified from "Interstitial Lung Disease in Connective Tissue Disorders" by A. Fischer and R. du Bois. Lancet 2012; 380: 689-98).

associated with varying degrees of interstitial fibrosis. These histologic changes correspond to HRCT findings of reticulation and GGOs with little or no architectural distortion and honeycombing. In rheumatic diseases, the predominant type of ILD was found to be NISP and UIP [4-6]. It has been recognized that rheumatic disease associated UIP (RD-UIP) shows better prognosis than idiopathic UIP or idiopathic pulmonary fibrosis (IPF) [7]. Unlike idiopathic ILD where NSIP was found to show better survival than UIP, RD-NSIP and RD-UIP have shown similar prognosis $[7,8]$ except for RA-ILD. Thus, it would be worth looking for evidence of rheumatic diseases in patients newly diagnosed with IPF.

In this review, we will introduce the updated knowledge on epidemiology, genetics based pathogenesis, clinical characteristics, and treatment options of rheumatic disease associated lung involvement, focusing on RA, SSc, myositis, and SLE.

\section{Lung Involvement in Rheumatoid Arthritis (RA)}

The most striking feature of RA lung involvement is that almost all components of the lung structure are potential targets of injury [1]. Parenchymal diseases include ILD or nodules while airway diseases include but are not limited to bronchiectasis (BE), bronchiolitis (associated with air trapping), and bronchial wall thickening [9-11]. Pleural and vascular structures can also be affected. The prevalence of a specific form of lung disease varies depending on the population studied and the modalities used. When examined by HRCT, lung abnormalities have been found in $50-70 \%$ of unselected RA patients [9-11], among which ILD and BE are most common. However, these abnormalities are, very often, not associated with any symptoms $[10,11]$. The majority of lung diseases during the course of RA occur during the first 5 years after RA diagnosis $[12,13]$, with the airway disease being the earliest manifestation [14]. The presence of ILD calls for particular attention since it leads to significant morbidity and mortality [15]. Airway diseases such as BE frequently occur in RA, but clinically severe cases are rare [16].

\subsection{RA Associated ILD (RA-ILD)}

2.1.1. Epidemiology and Clinical Characteristics of RA-ILD. The prevalence of clinically significant RA-ILD occurs in about $10 \%$ of RA patients [17] and in up to $58 \%$ if subclinical RA-ILD is included $[9,13,18]$. RA-ILD can occur before and throughout the course after the diagnosis of RA: $34 \%$ of RA-ILD occurred within 1 year before or after RA diagnosis [19] and the risk of RA-ILD increased with RA duration [20, 21]. The lifetime risk of developing ILD has been estimated to be $7.7 \%$ in RA patients compared to $0.9 \%$ in general population [15]. Risk factors for RA-ILD identified in most studies include male gender, older age at onset, smoking, and high titer of rheumatoid factor (RF)/anti-citrullinated protein antibodies (ACPA) [22, 23] while RA duration and severity have been identified in some but not all studies $[20,21]$. Unlike other rheumatic diseases where NSIP is the predominant histopathologic pattern, UIP accounts for almost half of RA-ILD patients, followed by NSIP $[5,24]$. The clinical course of RA-ILD is highly variable, ranging from asymptomatic to rapidly progressive. Once clinically present, symptoms usually progress over time, albeit with different rates of progression depending on the histopathologic patterns of ILD and other clinical characteristics such as extent of disease and rate of pulmonary function decline. In general, UIP pattern, more extensive disease, and rapid decline of pulmonary function during follow-up were found to associate with poor prognosis $[23,25]$. The hazard ratio (HR) of death in patients with RA-ILD was 3 times that of patients without [15]. Five-year mortality rate ranges from $35 \%$ to $39 \%$ after ILD diagnosis $[15,19]$.

Acute exacerbation (AE), a distinct fatal condition with rapid deterioration of respiratory status initially recognized in IPF, has been reported in ILD of rheumatic diseases including RA-ILD [26]. Radiographically, it is characterized by new development of GGOs or consolidations superimposed on the underlying reticular abnormalities. In RA-ILD, this condition was associated with 2.5-fold increase of mortality showing $64 \%$ of them dying during initial exacerbation [27]. UIP, old age at ILD diagnosis, and methotrexate usage were associated with AE of RA-ILD.

UIP pattern in HRCT was found to show different clinical course from non-UIP RA-ILD [28]. The former was associated with more frequent hospitalization and oxygen therapy and rapid decline of pulmonary function [29]. When the median survival time for all RA-ILD subjects was 5 years, RA-UIP had a worse median survival time than RA-nonUIP (3.2 versus 6.6 years), which did not differ from that of IPF [28]. The high prevalence of UIP partly explains high mortality rate in patients with RA-ILD. 


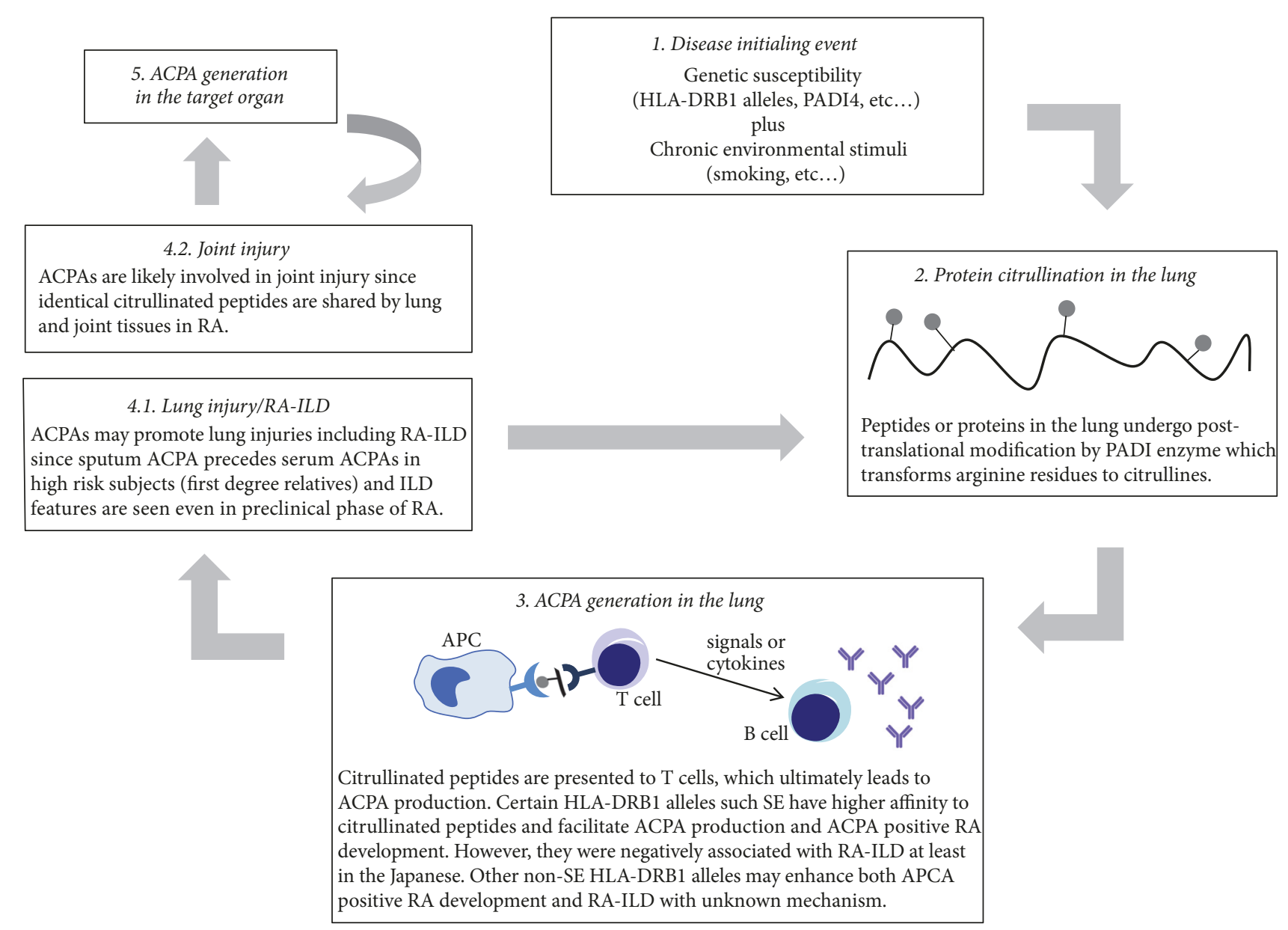

FIGURE 1: Schematic presentation of shared pathogenesis of RA and RA-ILD.

2.1.2. Genetics of RA and RA-ILD. Until now, the strongest genetic factor associated with RA has been shared epitope (SE) or certain HLA-DRB1 alleles that share a conserved amino acid sequence at positions 70-74 of the beta chain. These alleles were found to have higher binding affinity to citrullinated proteins than nascent proteins [30], which partly explains the underlying mechanism of association between RA specific antibodies or ACPAs and SE [31]. Smoking induces protein citrullination in the lung tissue [32]. The interaction between smoking and SE has been found to ultimately attribute to ACPA positive RA development [33]. This gene-environmental interaction is further supported by the gene-dose effect observed with anti-CCP positive RA development [33].

The strongest predictors of RA-ILD are RF and/or ACPA positivity and their higher titers correlate with more severe ILD [22]. Smoking has been a well-recognized risk factor not only for RA but also for RA-ILD [22]. RA and RA-ILD share common disease predictors (RF and ACPA) and an environmental risk factor (smoking) particularly affecting lung tissue. These findings may suggest that RA begins in the lung at least in smokers and that RA associated lung diseases including RA-ILD are the consequence of the same gene-environmental interaction as in RA: smoking may be a triggering insult to induce pulmonary APCA production that subsequently contributes to RA and RA-ILD development (Figure 1). Further findings that reinforce this hypothesis are as follows: (1) ACPA is locally produced in the lung tissue in early RA [34], (2) the pulmonary positivity of ACPAs or RF precedes serum positivity in high risk subjects [35], (3) ACPAs are associated with both ILD and airway disease in preclinical RA phase [36], (4) identical citrullinated peptides are shared in bronchial and synovial tissues in RA patients [37], and (5) a broader epitope is spreading in patients with RA-ILD than RA alone [38]. More compelling evidence to support this hypothesis has come from a study by Restrepo et al. where the association between RA-ILD and smoking was restricted in SE carriers [20].

However, the role of SE in RA-ILD pathogenesis seems to be more intriguing. Although SE has been shown to interact with an environmental risk factor to produce ACPA and the presence of ACPA is a strong predictor of RA-ILD, the association between SE and RA-ILD is not as straightforward as the association between SE and ACPA positive RA. SE was found to associate with deceased risk of RA-ILD in the Japanese [39]. Instead, HLA-DR2 serological group (HLADRB $1{ }^{*} 15$ and $\left.{ }^{*} 16\right)$ was associated with increased risk of RA-ILD $[39,40]$ and RA-UIP [41] in the Japanese. On the 
other hand, the distribution of SE was similar in RA versus RA-ILD in a UK population and there was an increased risk for RA-ILD by HLA-DRB1*07 [42]. The HLA-DRB1 alleles associated with RA-ILD might not be exactly SE alleles but they might also contribute to ACPA production. Likewise, each SE allele may differ in their roles in RAILD development although their roles in ACPA positive RA development are quite in the same direction. Recently, Song et al. explored polymorphisms in PADI4 and HLA-DRB1 in RA patients and found that recessive genotype of padi4_92 was associated with airway abnormalities and that tryptophan at position 9 of HLA-DRB1 amino acid sequence was strongly associated with RA-ILD in Korean patients with an odds ratio (OR) of 22.89 [43].

Although many RA susceptibility genes have been identified by genome wide association study (GWAS), few have been validated in RA-ILD subsets. A whole exome sequencing study has found that mutations in familial pulmonary fibrosis-linked genes (TERT, RTEL1, PARN, or SFTPC) are shared with RA-ILD [44].

2.1.3. Treatment of RA-ILD. The treatment for RA-ILD is quite empirical, because there have been no randomized placebo-controlled trials. Physicians generally follow the treatment strategies used for the corresponding type of idiopathic ILD. When encountered with AE of RA-ILD, moderate-to-high dose steroid therapy $(1 \mathrm{mg} / \mathrm{kg}$ oral prednisone or its equivalent) with or without another immunosuppressive agent is usually administered. Commonly used immunosuppressants in combination with steroids are azathioprine, cyclophosphamide (CYC), or mycophenolate mofetil (MMF). A recent study of 125 patients with RD-ILD receiving MMF, including 18 with RA-ILD, showed modest improvement in forced vital capacity (FVC) and diffusing capacity of carbon monoxide (DLCO) and reductions in prednisone dosage [45]. Rituximab treatment was successful for severe refractory RA-ILD in a small case series [46], whereas a recent single-center experience with rituximab for RD-ILD described inappreciable effect [47]. Perfenidone, an antifibrotic drug of unknown mechanism approved for IPF, might benefit RA-UIP phenotype, and studies are currently ongoing. Patients with non-UIP histopathologic patterns are more likely to respond to steroid/immunosuppressants. All patients with RA-ILD should be encouraged to cease smoking.

2.2. RA Associated Airway Diseases. Airway involvement is prevalent in $39-60 \%$ of patients with RA when assessed by HRCT $[10,13]$. Both upper and lower airways can be involved. The most common form of upper airway involvement is cricoarytenoid arthritis, found in nine out of fifteen RA patients by neck HRCT but only two by laryngoscopy, suggesting clinically significant involvement is infrequent [48]. Symptoms are variable including hoarseness, odynophagia, or dysphonia, but rarely prominent in most of the cases. However, it can cause upper airway obstruction, requiring immediate endotracheal intubation.

Among RA associated lower airway diseases, BE and bronchiolitis have long been recognized to associate with
RA $[49,50]$. BE refers to the permanent irreversible dilatation of cartilage-containing airways characterized by recurrent cough, sputum production, and respiratory infections. Abnormally dilated bronchi lead to impairment of host defenses, chronic bacterial infection, and airways inflammation, forming a vicious cycle [51]. A significantly higher prevalence $(2.7 \%)$ of symptomatic $\mathrm{BE}$ in RA patients has been noted compared to $0.03 \%$ in the general population [16]. HRCT detects BE at much higher frequencies in up to $30 \%$ of patients [10]. The temporal relationship between $\mathrm{BE}$ and RA has been a debate for a long time. BE far preceding RA has been reported in many studies while, in others, BE has been recognized as a late complication of RA [52, 53]. These conflicting reports are probably because most of the old studies rely on symptom onset of respiratory and articular systems. Recent HRCT studies have demonstrated a high prevalence of subclinical airway diseases resembling BE at the first diagnosis of RA or in preclinical RA $[14,54]$. Similar to ILD, high disease activity and autoantibody positivity were found to associate with BE in RA [55]. The coexistence of $\mathrm{RA}$ and $\mathrm{BE}$ has significant health implications in terms of infectious complications especially under treatment with disease modifying antirheumatic drugs (DMARDs). RA patients with $\mathrm{BE}$ have been shown to have mortality rates 7.3 times that of the general population, five times that of patients with RA alone, and 2.4 times that of patients with BE alone [56].

The mechanism for coexistence of RA and BE is unclear. A plausible hypothesis is that chronic bacterial infection as a result of $\mathrm{BE}$ provides a prolonged source of antigenic stimulation that leads to breakdown of immune tolerance and subsequent development of RA in genetically predisposed individuals [57]. The association of airway disease with RA seems to be different from that of RA-ILD. As in ILD, airway disease was found in preclinical RA [36]. However, RA patients with BE tended to be nonsmokers [58] and a high prevalence of BE (25\%) was identified in life-long nonsmoking RA patients [59]. Interestingly, SE was found to associate with airway disease in RA patients $[41,60]$. The shared genetic risk factors in terms of SE might contribute to the association between BE and RA. On the other hand, the RA-ILD predisposing allele HLADRB1 ${ }^{*}$ 15:02 was found protective against airway disease in Japanese RA [60]. HLA-DQB1 ${ }^{*}$ 03:01 and HLA-DQB1 ${ }^{*}$ 06:01 were found as susceptibility alleles for BE in RA [41, 61]. A polymorphism in PADI4 (recessive genotype of padi4_92) was reported to associate with the airway abnormalities in RA [43]. A small study showed that $16 \%$ of RA patients with diffuse BE were heterozygous for the delta F508 mutation of the cystic fibrosis transmembrane conductance regulator gene (CFTR) regardless of sweat chloride concentration or nasal potential difference measurements [62].

Follicular bronchiolitis and obliterative bronchiolitis (also referred to as constrictive bronchiolitis) are other lower airway involvements of RA than BE. Follicular bronchiolitis is pathologically defined as lymphoid hyperplasia with reactive germ cell centers within bronchiole walls [63]. Obliterative bronchiolitis is a fibrotic condition characterized by concentric narrowing of membranous and respiratory bronchioles caused by peribronchiolar inflammation and fibrosis without 
evidence of lymphoid hyperplasia. The prognosis of these conditions is reported to be poor [64].

2.3. RA Associated Pleural Involvement. Pleural abnormalities associated with RA include pleural effusion, pleuritis, pleural nodule, pneumothorax, and fibrothorax, among which pleuritis and pleural effusions are most common. Pleural disease used to represent the most common form of lung involvement in RA but has become far less prevalent in the last two decades probably due to early diagnosis of RA and more aggressive treatment. Although autopsy studies identified pleural effusion in up to 70\% [65], most of them are scanty and clinically silent. Pleural effusions in RA are exudative and sterile, often with mixed cell count (monocytes-predominant), high lactate dehydrogenase, low glucose, and low $\mathrm{pH}$ [65]. Most patients experience recovery along with DMARDs therapy.

2.4. RA Associated Vascular Disease. The representative vascular involvement in RA lung is rheumatoid vasculitis, characterized by destructive inflammatory infiltrate within smalland medium-sized blood vessel walls on pathology. However, primary vasculitic involvement of the lung is rare whereas peripheral neuropathy and cutaneous vasculitis are common. Pulmonary arterial hypertension (PAH) is extremely rare in RA.

\section{Lung Involvement in Systemic Sclerosis (SSc)}

SSc is a systemic autoimmune disease characterized by immune activation, vasculopathy, and fibrosis. Tissue fibrosis of the skin and internal organs is the hallmark of the disease that dictates the clinical course of SSc. Due to fibrosis and vasculopathy, the lung manifestation involves ILD and/or PAH, both of which are the leading causes of death, accounting for $33 \%$ and $28 \%$ of SSc associated mortality, respectively [66]. ILD and PAH can occur in both diffuse and limited subsets but in general, the former develops more frequently in the diffuse subset with anti-topoisomerase I antibody (ATA) and the latter in the limited subset with anti-centromere antibody (ACA). Other SSc-specific antibodies, anti-U3 RNP and antiTh/To antibodies, have shown heterogeneous associations with SSc-ILD [67]. Anti-U3 RNP antibodies were found to associate with ILD and PAH in a US study and with PAH in a UK study. Anti-Th/To antibodies were found to associate with SSc-ILD and PAH [67].

\subsection{SSc Associated ILD (SSc-ILD)}

3.1.1. Epidemiologic and Clinical Characteristics of SSC-ILD. It has been found that ILD is seen in up to $90 \%$ of patients with SSc depending on modalities used and patient populations studied [68]. HRCT has been considered as the gold standard detection method particularly compared to pulmonary function test, with the latter resulting in high false negative rate in early SSc-ILD: when FVC $<80 \%$ was used as a standalone method, $63 \%$ of SSc-ILD cases were undetected [69].
According to the analysis of the European Scleroderma Trials and Research group (EUSTAR) on 3656 patients with SSc, ILD was found in $53 \%$ in a diffuse and $35 \%$ in a limited subset by plain chest radiography [70]. Approximately $40 \%$ of patients with SSc were shown to experience moderate (FVC of $50-75 \%)$ to severe (FVC $<50 \%$ ) restrictive lung disease [71], with the latter being critically associated with mortality rate of $42 \%$ within 10 years after the onset of first non-Raynaud symptom.

The clinical course in terms of lung function change has been considered highly variable in SSc-ILD [72]: when 226 SSc patients with a median disease duration of 1.6 years were followed up for median 57 months, patients (51\%) with initial $\mathrm{FVC} \geq 80 \%$ rarely showed decline, while the others (49\%) with initial FVC $<80 \%$ were stable $(20 \%)$, deteriorated at various rates (16\%), or even improved (14\%) during 72 months of estimable trajectory time. Although highly variable, there are several consistent patterns for the clinical course of SSc-ILD. Most of the lung function decline occurs during the first 3-4 years after the onset of nonRaynaud's symptom, after which the decline is rather indolent $[71,73]$. The progression has been found to be best predicted by the low baseline FVC or higher extent of radiographic lung fibrosis [71-76]. Patients with normal HRCT or pulmonary functions at the time of diagnosis rarely develop ILD in the next 5 years. Other predictors of progression in some but not all studies were African-American ethnicity, cardiac involvement, male gender, early disease, abnormal nailfold capillaroscopy pattern, ATA positivity, and smoking [71-74, 77-79].

On the other hand, it has been consistently reported in a number of cross-sectional studies that esophageal dilation in HRCT is associated with more severe pulmonary function impairments [80-82]. The degree of esophageal diameter was found to negatively correlate with FVC and DLCO values [80]. Moreover, longitudinal correlations have been reported between the presence of high degree of gastroesophageal reflux (GER) and more rapid decline of pulmonary function values $[81,82]$. Notably, centrilobular fibrosis and bronchocentric distribution of lung involvement were found to be very common in SSc-ILD (84\% of all NSIP) and they were almost invariably associated with esophageal abnormalities [83]. This finding strongly suggests that microaspiration is one of the potential culprits to cause SSc-ILD progression. The issue whether GER has a deleterious effect on SScILD progression is of high importance since effective antiacid and/or anti-reflux therapy is available. However, both GER and SSc-ILD occur as early complications of SSc, which makes it difficult to assess their temporality for causal relationship. When examined in patients with very early diagnosis of SSc (VEDOSS), abnormal findings of esophageal involvement were prevalent with $75 \%$ of them showing abnormal pressure and speed of esophageal peristalsis [84]. Moreover, the association between esophageal involvement and positive lung HRCT or low DLCO values was observed as early as in patients with VEDOSS. GER and microaspiration have been proposed as pathogenic in IPF [85]. However, the causal mechanism can work in both directions with one in which IPF develops due to reflux of gastric contents and with 
the other in which GER is a consequence of distortion of mediastinal structure and greater transdiaphragmatic pressure gradient caused by IPF. Regardless of the true first trigger, a vicious cycle is created leading to accelerated lung injury, which also applies to SSc-ILD in a similar manner. To date, no prospective randomized trial has been done to address if GER treatment improves clinical outcomes of IPF. Two post hoc analyses on the placebo groups of patients with IPF from clinical trials reported contrasting results on proton pump inhibitors [86, 87]. Until now, it is not clear whether antacid treatment helps lung function preservation in IPF or ILD.

Histologic pattern of ILD was not associated with any clinical outcome [4,7], reflecting that the extent of lung involvement and degree of pulmonary function impairment are more relevant [72-76]. The predominant pattern of SScILD is NSIP based on both biopsy and HRCT [4, 68]. Although GGOs are often seen, the reversal is rarely observed during treatment, in less than $5 \%$ of the cases [88]. This finding suggests that the GGOs observed in SSc-ILD do, in fact, represent fine fibrosis compared to inflammation due to limited resolution of HRCT and are consistent with the result of the previous study where fibrotic rather than cellular NSIP constitutes the majority of SSc-NSIP (47/62) [4]. This concept is further supported by the finding that GGOs are replaced by fibrosis (honeycombing, bronchiectasis, and reticulation) regardless of therapy [89]. UIP patterns contribute to $15 \%$ of SSc-ILD but with more germinal centers and less fibroblastic foci [90], partly explaining that rheumatic disease associated UIP shows better survival than IPF.

The mortality of SSc patient, when evaluated by a metaanalysis [91], showed an overall threefold increase of standardized mortality rate: the 5 -year and 10-year survival of SSc patients from diagnosis were reported to be $74.9 \%$ and $62.5 \%$, respectively. The mortality risk among SSc patients with ILD was 2.89 times compared to those without.

3.1.2. Genetics of SSc and SSc-ILD. Since the first large scale GWAS performed on SSc patients in 2010 [92], many SSc susceptibility genes within and outside of the major histocompatibility complex (MHC) region have been identified by subsequent GWAS, immunochips, and their follow-up studies [93]. MHC class II region has been the most significant susceptibility locus while non-MHC genes continue to be identified. One of the prominent features of SSc susceptibility genes outside MHC is that vast majority of them are in fact susceptibility genes of other autoimmune diseases such as SLE, RA, multiple sclerosis, inflammatory bowel disease, and primary biliary cirrhosis (PBC) [93]. When autoimmune diseases are clustered based on the number of shared susceptibility genes at GWAS level, SSc was found to most closely correlate with SLE and PBC [93].

The SSc susceptibility genes can be classified according to their roles: those involved in immune functions and inflammation and those involved in extracellular matrix (ECM) deposition and fibrosis. The functions of the former genes include autophagy/apoptosis and DNA clearance (ATG5, PPARG, FAS, RHOB, and DNASE1L3), type I interferon (IFN) signaling (IRF5, IRF7, and IRF8), IL12 signaling (IL12A, IL12RB2, IL12RB1, and STAT4), other cytokines and signaling (TNIP1, TNFAIP3, IRAK1, and TNFSF4), and adaptive immunity of B (BANK1, BLK) and T cells (CD247, CSK, and PTPN22) [94]. Although the hallmark of SSc is fibrosis, the number of GWAS genes (e.g., PPARG) directly involved in fibrosis is far less than those in immune system and inflammation. This finding partly reflects study design driven bias associated with the use of immunochips. However, the paucity of fibrosis genes found by GWAS or GWAS followup studies raises several scenarios: tissue fibrosis in SSc might be (1) the downstream result of immune activation and inflammation, (2) effect size failing to reach statistical significance at GWAS level, (3) associated with rare genetic variants, or (4) the result of epigenetics. Since treatment effect with immunosuppressants has been limited against skin or lung fibrosis mainly stabilizing rather than reversing it $[95,96]$, the first explanation looks skeptical. Candidate gene analyses, which might have advantage to capture genes with small effect size, have revealed fibrosis related genes, caveolin-1 gene and CTGF, as SSc susceptibility genes [97, 98]. A recent approach using whole exome sequencing seems to partly complement GWAS-based genetics by detecting rare coding variants involved in fibrosis. Using this method, novel SSc susceptibility genes enriched in ECM related pathway (COL4A3, COL4A4, COL5A2, COL13A1, and COL22A1) and SSc-ILD susceptibility gene (XRCC4) involved in DNA repair have been further identified [99]. Since the concordance rate between monozygotic twins is only $4 \%$ in SSc, one can easily expect that, in addition to genetic factors, epigenetic alterations specific to genes, cells, and tissues play an important role in SSc. Epigenetic mechanisms include DNA methylation, histone modification, and noncoding RNAs including miRNAs. Evidence is rapidly accumulating that these mechanisms are distinctively used among immune cells and tissue fibroblasts of SSc patients $[100,101]$. Epigenetics in SSc will not be discussed here.

Among the SSc susceptibility genes identified by GWAS and candidate gene analyses, only a limited number of genes were investigated about their association with SSc-ILD. The IFN regulatory factor 5 gene (IRF5) encodes one of the IFN regulatory factors critical for type I IFN regulation and virus-induced immune activation. Recently, IRF5 rs2004640 $\mathrm{T}$ allele (also known to be associated with SLE), which creates a donor splice site in intron 1 of IRF5 leading to transcription of the alternative exon $1 \mathrm{~B}$, was found to be associated with SSc and SSc-ILD in a European French population [102]. IRF5 rs4728142 A allele was found to be associated with lower IRF5 expression, higher FVC at enrollment, and better survival in Caucasian SSc patients [103]. STAT4 rs757486 T allele and IRF5 rs2004640 $\mathrm{T}$ allele were shown to have an additive effect towards susceptibility to SSc-ILD [104]. ALOX5AP rs10507391 A allele was also found to have an association with SSc-ILD in a European population enrolled in an EUSTAR group [105]. Other genes whose polymorphisms were shown to associate with SSc-ILD include CTGF [97, 98], NLRP1 (also having an additive risk with IFR5 and STAT4 on SScILD) [106], CD226 [107], and HGF [108].

Ironically, one of the most striking features of SSc-ILD genetics has come from the IPF gene studies. None of the non-MHC susceptibility genes found by IPF GWAS were 
associated with SSc-ILD, which contrasts the distinctive pathogenesis of SSc-ILD and IPF [109-112].

3.1.3. Treatment of SSc-ILD. Since those with initial FVC $\geq$ $80 \%$ rarely show decline in lung function [72], treatments should be focused on symptomatic patients with moderate to severe extent or with progression. As mentioned in the previous section, GGOs found in HRCT of SSc-ILD patients may represent fine fibrosis rather than inflammation $[88,89]$. In line with this finding, the main effect of anti-inflammatory or immunosuppressive treatment against SSc-ILD has been stabilizing lung function rather than improving it. In the Scleroderma Lung Study (SLS) I, 158 SSc patients who had symptomatic ILD with an evidence of active alveolitis and a FVC between 45 and $85 \%$ were randomly allocated to oral CYC versus placebo for one year and were followed for another year [95]. The mean absolute FVC difference of $2.53 \%$ at 12 months was significant in favor of CYC. The effect was maintained at 1 year off treatment with the mean absolute FVC difference of $1.95 \%$ but disappeared by 2 years off treatment [113]. In SLS II where MMF for 2 years was compared with oral CYC for one year, the two drugs showed similar results at 2 years with better safety profile for MMF [96]. Their mean FVC improved from baseline by $2.17 \%$ in MMF group and 2.86\% in CYC group. However, 2017 updated EULAR recommendation still suggests cyclophosphamide preferentially over MMF based on two previous high-quality randomized controlled trials consistently showing an efficacy of CYC against SSc-ILD compared to placebo [95, 114]. MMF could be considered as a first-line treatment for patients with comorbidities and little tolerability to toxicities. In addition to traditional immunosuppressives, both tocilizumab (IL-6 receptor antagonist) and rituximab (B cell depleting agent) have shown efficacy in SSc-ILD and further investigations are underway $[115,116]$. One of the tyrosine kinase inhibitors, nintedanib, affects receptor signaling of VEGF, PDGF, and FGF. It has shown efficacy against IPF and is under phase III trial for SSc-ILD. Combination treatment with pirfenidone and MMF is now being investigated for SSc-ILD.

3.2. SSc Associated Pulmonary Arterial Hypertension (SSc$P A H)$. The pathogenic mechanisms underlying $\mathrm{PAH}$ are abnormal proliferation, vasoconstriction, and thrombosis of pulmonary vasculature. The presence of $\mathrm{PAH}$ is defined at right heart catheterization (RHC) by a mean pulmonary arterial pressure of $\geq 25 \mathrm{~mm} \mathrm{Hg}$ with a pulmonary capillary wedge pressure of $\leq 15 \mathrm{~mm} \mathrm{Hg}$. However, all forms of pulmonary hypertension can occur in SSc: isolated $\mathrm{PAH}$, pulmonary hypertension from left heart dysfunction, and pulmonary hypertension secondary to ILD or hypoxia. Moreover, combinations of these forms can occur in SSc. However, this review will focus on isolated PAH.

3.2.1. Epidemiologic and Clinical Characteristics of SSc-PAH. $\mathrm{PAH}$ is a serious complication of SSc with high mortality if not promptly diagnosed and properly treated. The prevalence of SSc-PAH varies depending on the method used and populations studied. When assessed by RHC in high risk patients identified by echocardiography, DLCO patterns, or unexplained dyspnea, PAH affects $8-12 \%$ of SSc patients $[117,118]$. SSc-PAH is more common in a limited than diffuse subset disease. In particular, SSc-PAH has been observed in up to $50 \%$ of CREST syndrome [119]. Unlike SSc-ILD which is an early complication of SSc in most cases, SSc-PAH occurs within 5 years from the first non-Raynaud phenomenon symptom in half of the cases and the mean interval between SSc diagnoses and PAH occurrence was 6.3 years [120].

As reflected in a study where active surveillance and early treatment of PAH improve survival compared to passive identification during routine practice [121], the poor prognosis of SSc-PAH is partly attributed to delayed diagnosis due to clinically silent nature of the disease until advanced. SScspecific risk factors for $\mathrm{PAH}$ include male gender, old age, presence of ACA or anti-U3 RNP, presence of telangiectasia, digital ulcers, and limited subset/CREST syndrome [122]. However, none of these risk factors are sufficient indicators of PAH. The laboratory findings highly suggesting $\mathrm{PAH}$ include elevated levels of $\mathrm{N}$-terminal probrain natriuretic peptide (NT-proBNP) or disproportionate decrease of DLCO [123-125]. Since RHC is invasive, echocardiographic measure of tricuspid regurgitation (TR) velocity is often used as a screening tool to select patient candidates for RHC and TR velocity $>2.5 \mathrm{~m} / \mathrm{s}$ has been considered as a threshold to suspect PAH. However, $20 \%$ of high risk patients with mild SSc-PAH were not detected at this threshold [126] and the sensitivity of echocardiography has ranged from $50 \%$ to $90 \%[127,128]$. The DETECT study proposed an algorithm to select patients under high suspicion of PAH for referral to RHC and to minimize missed or delayed diagnoses [126] (Figure 2). When compared with European Society of Cardiology/European Respiratory Society 2009 guidelines, the DETECT algorithm recommended more patients for RHC and detected more patients with PAH [129]. In DETECT study where 57 patients with SSc-PAH were followed [130], $44 \%(25 / 57)$ of them showed progression during a median follow-up of 12.6 months. Thirteen of the twenty-five showed mild PAH in WHO functional class I or II. Factors associated with progression were male gender, lower DLCO, higher FVC/DLCO ratio, and poor functional capacity [130].

The adjusted survival was significantly worse in ILD associated PAH than in SSc-PAH showing a 5-fold increase of mortality risk: 3-year survival rates were 39\% in ILD$\mathrm{PAH}$ versus $64 \%$ in SSc-PAH [131]. The overall survival in SSc-PAH has been reported as $81 \%, 64 \%$, and $52 \%$ at 1 year, 2 years, and 3 years, respectively, in a meta-analysis [132]. Compared to SSc patients without PAH, patients with SSc$\mathrm{PAH}$ showed more than threefold increase of mortality risk [133]. The prognosis of SSc-PAH has been found to be worst compared to idiopathic or PAH of other rheumatic diseases [134].

3.2.2. Genetics of SSc-PAH. Genes identified for the risk of $\mathrm{SSc}$ as well as those implicated in idiopathic PAH have been pursued by large scale case-control studies using a candidate gene approach. MIF rs755622 C allele in the promoter region was associated with SSc-PAH particularly in the diffuse 
Two-step decision tree of DETECT algorithm

\section{Step 1.}

Non-echocardiographic variables

(i) $\mathrm{FVC} \%$ predicted/DLCO $\%$ predicted

(ii) Current or past telangiectasia

(iii) Serum ACA

(iv) Serum NT-proBNP level

(v) Serum urate level

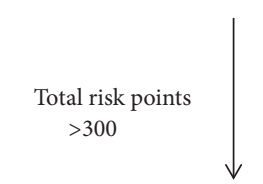

Step 2.

Total risk points from Step 1 plus

echocardiographic variables

(i) Right atrium area

(ii) TR velocity

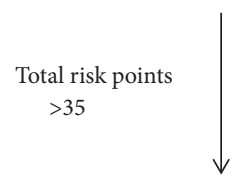

Right heart catheterization
Normograms for practical application of DETECT algorithm

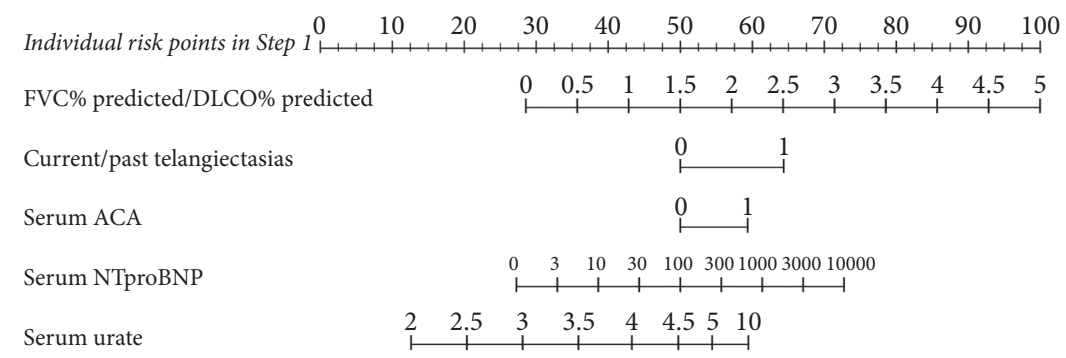

ECG: right axis deviation

Total risk points from Step 1

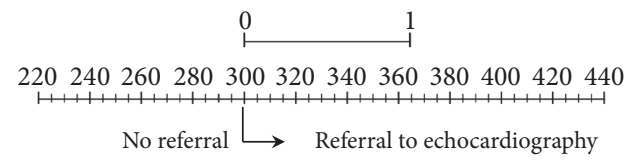

\begin{tabular}{|c|c|c|c|c|c|c|c|c|}
\hline Individual risk points in Step 2 & 10 & & & 30 & 4 & & 50 & 60 \\
\hline Total & 300 & 320 & 340 & 36 & 380 & 400 & 420 & \\
\hline
\end{tabular}

Right atrium area

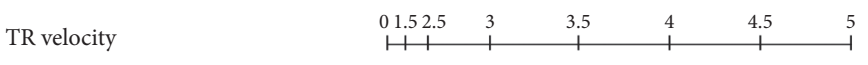

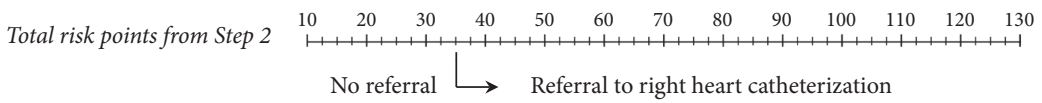

FIGURE 2: DETECT algorithm. ACA: anti-centromere antibody; DLCO: diffusing capacity of carbon monoxide; FVC: forced vital capacity; NT-proBNP: N-terminal probrain natriuretic peptide; TR: tricuspid regurgitation (cited and modified from "Evidence-Based Detection of Pulmonary Arterial Hypertension in Systemic Sclerosis: The DETECT Study” by Coghlan JG, et al. Ann Rheum Dis 2014; 73: 1340-9).

subset phenotype combined from multiple cohorts of European origin [135]. The SSc susceptibility gene PPARG rs10865710 C allele showed an association with SSc-PAH in a French population [136]. KCNA 5, another SSc susceptibility gene, is implicated in vascular tone regulation that its inhibition during hypoxia produces pulmonary vasoconstriction. Its variants showed conflicting result with an association with SSc-PAH in a French population [137] but failed to be replicated in other European ancestries [138]. A rare nonsynonymous TLR2 variant (Pro63His) was found to be associated with ATA positivity, diffuse subset, and increased risk of SScPAH development $(\mathrm{HR}=5.61)$ in a European population of multiple origins [139]. This variant was found to increase the levels of IL- 6 and TNF- $\alpha$. UPAR encodes a pleiotropic receptor involved in fibrosis and vascular remodeling and its rs344781 $\mathrm{G}$ allele was associated with limited subset, digital ulcers, and SSc-PAH in a combined cohort of Italian and French origins [140]. G allele of rs5029939 at TNFAIP3 encoding ubiquitin-modifying enzyme has been shown to associate with diffuse subset $(\mathrm{OR}=2.71)$, SSc-ILD $(\mathrm{OR}=$ $2.26)$, and SSc-PAH $(\mathrm{OR}=3.11)$ in a European population of multiple origins [141]. Two SNPs at IL23R, rs11209026 G allele and rs11465804 $\mathrm{T}$ allele, showed positive associations with diffuse subset and ATA positivity and negative association with SSc-PAH in a US population [142]. The CXC chemokine stromal cell-derived factor 1 (SDF-1/CXCL12) and its receptor CXCR4 are involved in regulation of angiogenesis. SDF1- $3^{\prime} \mathrm{A}$ allele was associated with SSc-PAH $(\mathrm{OR}=2.37)$ and digital ulcers $(\mathrm{OR}=2.33)$ [143].

3.2.3. Treatment of SSc-PAH. Active surveillance and early treatment may improve survival in SSc-PAH [121]. According to 2013 American College of Rheumatology recommendations for screening and monitoring of PAH in rheumatic diseases [144], all SSc patients, patients having SSc spectrum disorders (having sclerodactyly, nailfold capillary abnormalities, or SSc-specific autoantibodies), or those with $\mathrm{PAH}$ signs/symptoms (dyspnea on rest/exercise, fatigue, presyncope/syncope, chest pain, palpitations, dizziness, and lightheadedness) should undergo screening evaluations for $\mathrm{PAH}$ using PFTs with DLCO, echocardiography, and NT-proBNP. The panel also endorsed DETECT algorithm in SSc patients [126] if DLCO $<60 \%$ and SSc duration $>3$ years from the first non-Raynaud's symptom. The recommendation criteria for RHC in these patients are shown in Table 2 [144]. In SSc and SSc spectrum disorders, annual follow-up of echocardiography and PFTs was recommended. The full screening panel (echocardiography, PFT, and NT-proBNP) should be performed as soon as any new signs or symptoms are present. The diagnosis of PAH should reply on RHC. 
TABLE 2: Recommendations for right heart catheterization for SSc and SSc-spectrum disorder.

\begin{tabular}{|c|c|c|}
\hline Modalities & Parameter thresholds required for RHC & $\begin{array}{l}\text { Signs/symptoms* } \\
\text { required for RHC }\end{array}$ \\
\hline \multirow{6}{*}{ Echocardiography } & TR velocity & \\
\hline & $2.5-2.8 \mathrm{~m} / \mathrm{s}$ & Yes \\
\hline & $>2.8 \mathrm{~m} / \mathrm{s}$ & No \\
\hline & Cavity enlargements irrespective of TR velocity & \\
\hline & Right atrial major dimension $>53 \mathrm{~mm}$ or & No \\
\hline & Right ventricular mid-cavity dimension $>35 \mathrm{~mm}$ & \\
\hline \multirow[b]{2}{*}{$\begin{array}{l}\text { Pulmonary function } \\
\text { tests }\end{array}$} & FVC/DLCO ratio $>1.6$ and/or DLCO $<60 \%^{* *}$ & Yes \\
\hline & $\begin{array}{l}\text { FVC/DLCO ratio }>1.6 \text { and/or DLCO }<60 \% \text { and NT-pro BNP }>2 \text { times upper } \\
\text { limit of normal }\end{array}$ & No \\
\hline Composite measures & $\begin{array}{l}\text { Meets DETECT algorithm in patients with DLCO }<60 \% \text { and disease duration of } \\
>3 \text { years from 1st non-Raynaud's symptom }\end{array}$ & No \\
\hline
\end{tabular}

*Symptoms: dyspnea on rest or exercise, fatigue, presyncope/syncope, chest pain, palpitations, dizziness, and lightheadedness. Signs: loud pulmonic sound and peripheral edema. ${ }^{* *}$ Without overt systolic dysfunction, greater than grade I diastolic dysfunction or greater than mild mitral or aortic valve disease, or evidence of PAH in echocardiography; DLCO: diffusing capacity of carbon monoxide; FVC: forced vital capacity; NT-proBNP: N-terminal probrain natriuretic peptide; PAH: pulmonary arterial hypertension; SSc: systemic sclerosis; TR: tricuspid regurgitation (cited and modified from "Recommendations for Screening and Detection of Connective Tissue Disease-Associated Pulmonary Arterial Hypertension" by D. Khanna, C. H. Tseng, N. Farmani et al. Arthritis Rheum 2013; 65: 3194-201).

Vasodilating calcium channel blockers are used as the first-line treatment for SSc-ILD, but for most of the progressive cases, they are insufficient to resolve symptoms and $\mathrm{PAH}$ related hemodynamics. Systemic prostacyclin analogues have been used and more recently, endothelin-1 receptor antagonists and phosphodiesterase type 5 inhibitors have been used to treat PAH [145]. However, these treatments do not show survival benefits to date beyond intermediary outcomes such as symptom improvement and exercise tolerance. Among $\mathrm{PAH}$ of WHO group 1, SSc-PAH is generally the least responsive to therapy and has a significant mortality [134, 145].

Nevertheless, the results of several recent randomized controlled studies are promising and worth to mention. The SERAPHIN trial compared the efficacy of the long-term treatment with nonselective endothelin receptor antagonist, macitentan versus placebo in symptomatic PAH including $\mathrm{RD}-\mathrm{PAH}$. After 3 years, macitentan reduced the risk of composite end point for death due to $\mathrm{PAH}$ and worsening of $\mathrm{PAH}$ in a dose dependent manner by $30 \%$ with $3 \mathrm{mg}$ treatment and $50 \%$ with $10 \mathrm{mg}$ treatment [146]. The effect was consistent for RD-PAH in subgroup analysis and was significant even in those receiving background treatment with phosphodiesterase type 5 inhibitors. The AMBITION trial, comparing ambrisentan (endothelin receptor A selective antagonist) plus tadalafil (phosphodiesterase type 5 inhibitor) versus either ambrisentan or tadalafil monotherapy in treatment naive symptomatic $\mathrm{PAH}$, showed that the combination therapy reduced the risk of composite outcome of death of any cause and worsening of PAH by approximately $50 \%$ [147]. The post hoc analysis showed that this effect was consistent in both subgroups of RD-PAH and SSc-PAH [148]. In the phase 2 GRIPHON trial, selexipag (oral selective IP prostacyclin receptor agonist structurally distinct from prostacyclin) reduced the risk of composite endpoint of death or a complication related to PAH compared to placebo, with similar efficacy regardless of baseline PAH therapy [149]. Post hoc analysis on RD-PAH patients (half from SSc-PAH) from the GRIPHON trial showed $41 \%$ risk reduction for the same composite endpoint in RD-PAH and SSc-PAH [150]. These clinical trials not only show the efficacy of updated vasodilatory drugs but also imply that combination of drugs with different vasodilating mechanisms is also effective. Going one step forward, combination therapy targeting all three components of disease pathogenesis (vasculopathy, fibrosis, and autoimmunity) may be tried to improve prognosis.

\section{Lung Involvement in Myositis}

In the majority of cases, myositis-associated lung involvement takes a form of ILD. Unlike SSc, PAH is often secondary to ILD and isolated PAH is rarely found [151]. Although not a primary lung involvement of myositis, aspiration pneumonia and hypoventilatory respiratory failure are two serious complications related to pharyngeal and respiratory muscle involvement, respectively. The review will focus on myositis-associated ILD.

4.1. Epidemiologic and Clinical Characteristics of MyositisAssociated ILD. The prevalence of ILD ranges from 23.1 to $65 \%$ in patients with myositis depending on the modalities used $[152,153]$. However, when associated with antiaminoacyl tRNA synthetase (ARS) antibody syndrome, the prevalence exceeds 70\% [154]. As in ILD of other rheumatic diseases, ILD can appear with, before, or after the onset of skin or muscle manifestations but tends to be a component of early myositis [152, 155]. Although the clinical course of myositis-associated ILD is variable, it is distinguished from SSc-ILD in that the former can be categorized in general into three clinical patterns based on respiratory symptoms at presentation $[152,156]$ : rapidly progressive form with acute onset symptoms, chronic form with slowly progressive 
symptoms, and asymptomatic or subclinical form. This is in contrast to SSc-ILD where fibrosis is the hallmark of the disease and progression occurs in a more chronic way. This difference reflects that inflammatory change is more prominent in myositis-associated ILD, generally in proportion to the rate of symptom deterioration, being most severe in rapidly progressive form where respiratory failure occurs within weeks [156].

Chronic form presenting with insidious onset dyspnea and a nonproductive cough is the most common variant (50\%) and rarely shows constitutional symptoms. Up to $30 \%$ of polymyositis (PM) and dermatomyositis (DM) patients seem to have subclinical or asymptomatic ILD $[152,157]$. This lack of overt symptoms emphasizes the need for pulmonary screening in all myositis patients, especially those with antiJo-1 antibody (=anti-histidyl tRNA synthetase antibody). However, ILD that initially presents as the aforementioned two patterns can transform into the rapidly progressive pattern during the later course of the disease. The rapidly progressive forms often take a histopathologic finding of diffuse alveolar damage (DAD) and occur in less than $20 \%$ of PM and DM patients with ILD [152] and are often accompanied by fever and malaise. The occurrence of rapidly progressive ILD has been well noted in patients with socalled amyopathic dermatomyositis (ADM) who have the typical rash of DM (Gottron's papules and/or heliotrope rash) but without muscle symptoms $[157,158]$. ILD in these patients characteristically responds poorly to even aggressive treatment and shows high mortality rate [157]. Overall 5-year survival rates of patients with myositis-associated ILD are around $70 \%[6,152]$.

Although NSIP is the most predominant histologic pattern followed by UIP $[6,63,154]$, other histologic patterns have been frequently observed in patients with myositisassociated ILD, including COP and DAD [159]. Furthermore, ILD in myositis tends to exhibit a mixture of more than one histological pattern [63]. Treatment response of ILD varies depending on the underlying histological pattern [159]. COP responds favorably to steroids, whereas DAD and UIP respond poorly to immunosuppressive therapies with poor prognoses $[152,159]$. The response of NSIP to steroids depends on degrees of inflammation and fibrosis $[6,63]$.

As in SSc, myositis specific autoantibodies tend to be mutually exclusive and are associated with distinctive clinical subsets. Other than anti-ARS antibodies, anti-melanoma differentiation-associated gene 5 (anti-MDA5) antibodies are associated with rapidly progressive ILD and/or ADM [160, 161]. Anti-ARS associated ILD shows more chronic course than anti-MDA5 associated ILD [161].

4.2. Genetics of Myositis and Myositis-Associated ILD. The two GWAS in myositis of European ancestry have shown that the strongest peak in Manhattan plot resides in MHC region of HLA 8.1 ancestral haplotype $[162,163]$. Other suggestive genes outside of MHC region were PLCL1, BLK, and CCL21 in DM [163]. A GWAS imputation study also revealed TYK2 and FAM167A-BLK region as a susceptibility locus in DM [164]. FAM167A-BLK polymorphism was confirmed in Japanese myositis patients [165] and in Chinese patients with myositis or myositis-ILD [166]. A large scale immunochip study has shown that PTPN22, UBE2L3, CD28, TRAF6, and STAT4 are associated with myositis of Caucasian descents [167]: PTPN22 was primarily associated with PM. Other suggestive associations were IL18R1 and RGS1 in PM and GSDMB in DM. STAT4 had been shown to associate with myositis in a Japanese population [168].

Only a limited number of genes were examined against ILD phenotype. In large scale case-control studies in a Chinese Han population using candidate gene approach, CCL21, a myositis susceptibility gene in Caucasians [163], was found to associate with PM or PM-associated ILD [169], TNFAIP3 and IRF5 with myositis or myositis-associated ILD [170], and PLCL1 with DM and DM-associated ILD [171].

4.3. Treatment of Myositis-Associated ILD. High dose steroid therapy has been the primary treatment for myositisassociated ILD. The responsive rate against steroid monotherapy has been reported to be $50 \%$ [156]. Nowadays, various immunosuppressives are being used to treat myositisassociated ILD in combination with steroids, such as CYC, cyclosporine, or MMF. However, the drug effectiveness has been typically reported in retrospective observational studies of small sample size and/or case series without randomized controlled trials. Retrospective uncontrolled studies have reported effectiveness of rituximab in the setting of ILD $[46,172]$. Tocilizumab, abatacept, or sifalimumab are under investigation for their efficacy in myositis [173].

\section{Lung Involvement in Systemic Lupus Erythematosus (SLE)}

The prevalence of lung involvement varies depending on the population and the detection method, as in other rheumatic diseases. Pulmonary function and HRCT abnormalities are common [174-176], but many are asymptomatic $[174,176]$. As in RA, pulmonary manifestations related to SLE can affect any component of the lung structure from pleura, vasculature, parenchyma, and airways [174-176].

5.1. SLE Associated Pleural Disease. Pleural involvement constitutes one of the classification criteria of SLE. Clinically apparent pleural effusions have been observed in up to $50 \%$ of SLE patients with a prevalence of up to 93\% at necropsy [177]. Effusions are either bilateral or unilateral. The pleural fluids in lupus pleuritis are often exudative with increased leukocyte cell count (polymorphonuclear neutrophils or lymphocytes predominant). The glucose level could be either normal or low as in lupus pleuritis [178]. Checking antinuclear antibodies (ANAs) of pleural fluid may be helpful for differential diagnosis of effusions, and a high titer of ANA $(\geq 1 / 160)$ in pleural fluid is strongly indicative of SLE pleuritis [179]. SLE pleuritis responds favorably to NSAIDs for mild cases. For moderate to severe effusions, oral steroids are generally effective. Systemic immunosuppressive agents are infrequently indicated. 
Shrinking lung syndrome is a rare manifestation of SLE, characterized by dyspnea, pleuritic chest pain, and a progressive decrease in lung volumes as reflected in restrictive PFT or diaphragmatic elevation, with no parenchymal or pulmonary vascular lesions. The reported prevalence of shrinking lung syndrome is around $1 \%[180,181]$. The pathogenesis of shrinking lung syndrome is unclear but several hypothetical models have been proposed without confirming evidence: microatelectasis associated with decreased surfactants, respiratory muscle weakness, diaphragm fibrosis and phrenic nerve palsy, and pleural inflammation. In particular, the most recent hypothesis claims that pleural inflammation or pleuritis has been proposed to cause diaphragmatic dysfunction with chronic hypoinflation and subsequent lung remodeling towards loss of compliance [182]. Although there are no standardized treatment regimens of shrinking lung syndrome, moderate-to-high dose steroids are used as firstline drugs with good success rates [181]. Immunosuppressives are combined if steroid treatment fails or from the beginning for severe cases. Successful use of rituximab in steroid-refractory cases has been consistently reported [181]. Theophylline and $\beta$-agonists have been tried to improve diaphragmatic strength, showing efficacy in $15 \%$ to $30 \%$ of patients. Overall, majority of patients show significant improvement in symptoms and PFT and long-term prognosis is favorable without resulting in respiratory failure or disease associated mortality [181].

5.2. SLE Associated Vascular Disease of the Lung. The vascular involvements of the lung in SLE patients include PAH and DAH. The prevalence of SLE associated PAH (SLE-PAH) has been typically around 4-5\% [183-185]. Anti-cardiolipin antibodies (or lupus anticoagulant), Raynaud's phenomenon, or anit-U1 RNP antibodies were found to associate with PAH in SLE [184-186]. One-year survival of SLE patients with PAH from REVEAL registry was $94 \%$, better than SScPAH (82\%) and similar to RA-PAH (96\%) [135]. Vasodilating treatment for SLE-PAH is similar to that for idiopathic PAH. However, additional immunosuppressive agents (steroids or CYC) seem to be beneficial in SLE-PAH [187-189].

DAH is a rare but devastating complication of SLE and its prevalence has been $<2 \%$ of patients with SLE [190]. The onset of DAH is abrupt and the symptoms usually develop over hours to a few days. DAH could be either the presenting feature or observed in known SLE patients during a generalized lupus flare with associated multisystem involvement [190]. The mortality may approach beyond 50\% $[190,191]$. Majority of patients are treated with high dose of steroids in combination with immunosuppressives with or without plasma exchange and/or intravenous immunoglobulin. Several case series have shown the successful treatment of SLE-DAH with CYC [191] or rituximab [192].

5.3. SLE Associated Parenchymal Disease. ILD is less common in SLE than in other CTDs, with clinically significant cases observed in less than $8 \%$ of SLE population [193]. NSIP appears to be most common, while UIP pattern is uncommon [24]. When ILD is active with symptoms or progression, high dose steroids and immunosuppressive agents such as azathioprine, CYC, or MMF could be used.

5.4. SLE Associated Airway Disease. Airway disease is uncommon manifestation in SLE. However, upper and lower airways are both potential targets. Upper airway disease may range from mild ulceration, vocal cord paralysis, cricoarytenoid arthritis, and necrotizing vasculitis with airway obstruction [194]. Coexistence of SLE and BE on chest HRCT has been reported, though its clinical significance is uncertain [174-176]. SLE cases with bronchiolitis obliterans and COP have been rarely described [177].

\section{Management Strategies on How to Screen and Monitor Lung Involvements in Rheumatic Diseases}

Figure 3 shows a schematic picture on when or how to screen and monitor patients with rheumatic disease associated lung involvements focusing on ILD and PAH. Due to the impact of lung involvements on the patient prognosis, meticulous history taking on respiratory symptoms and relevant physical examinations are mandatory at the time of diagnosis of each rheumatic disease. Noninvasive PFTs are often recommended at diagnosis regardless of respiratory symptoms based on high frequency of subclinical involvements. However, because of the considerable heterogeneities in onset, involved structural components, severity, and prognosis of rheumatic disease associated lung diseases, there is no clear consensus on when and how to screen and monitor them. Although many physicians perform chest HRCT usually upon new onset or worsening of respiratory symptoms, auscultatory Velcro rales or abnormalities on the chest radiograph or PFTs appear, and the use of chest HRCT at the time of diagnosis has been stressed upon by some experts based on the high prevalence and prognostic implications of lung parenchymal involvements, particularly ILD, in rheumatic diseases. However, due to radiation exposure and nonprogressive nature in a significant proportion of asymptomatic patients, the use of HRCT as mandatory initial work-up and/or for periodic monitoring is controversial despite its better performance in detecting ILD than PFTs. Thus, its use has been justified for those at high risk identified by symptoms and signs (Figure 3). Monitoring is done using PFTs with or without chest radiography at a frequency depending on the severity and progression rate of lung diseases. Annual follow-up would be sufficient for asymptomatic mild cases while monthly or even shorter follow-up is required for rapidly progressive cases. In case of $\mathrm{PAH}$, since symptoms are nonspecific and physical examination/imaging studies are not much helpful, early detection is difficult while early treatment would improve patient quality of life and prognosis. $\mathrm{PAH}$ should be screened using echocardiography, PFTs, and NT-proBNP in particular situations even asymptomatic, in case of SSc and SSc spectrum disorders (Table 2, Figure 3). Any rheumatic disease patients with PAH symptoms/signs should also be screened. Once patients show highly suggestive findings 


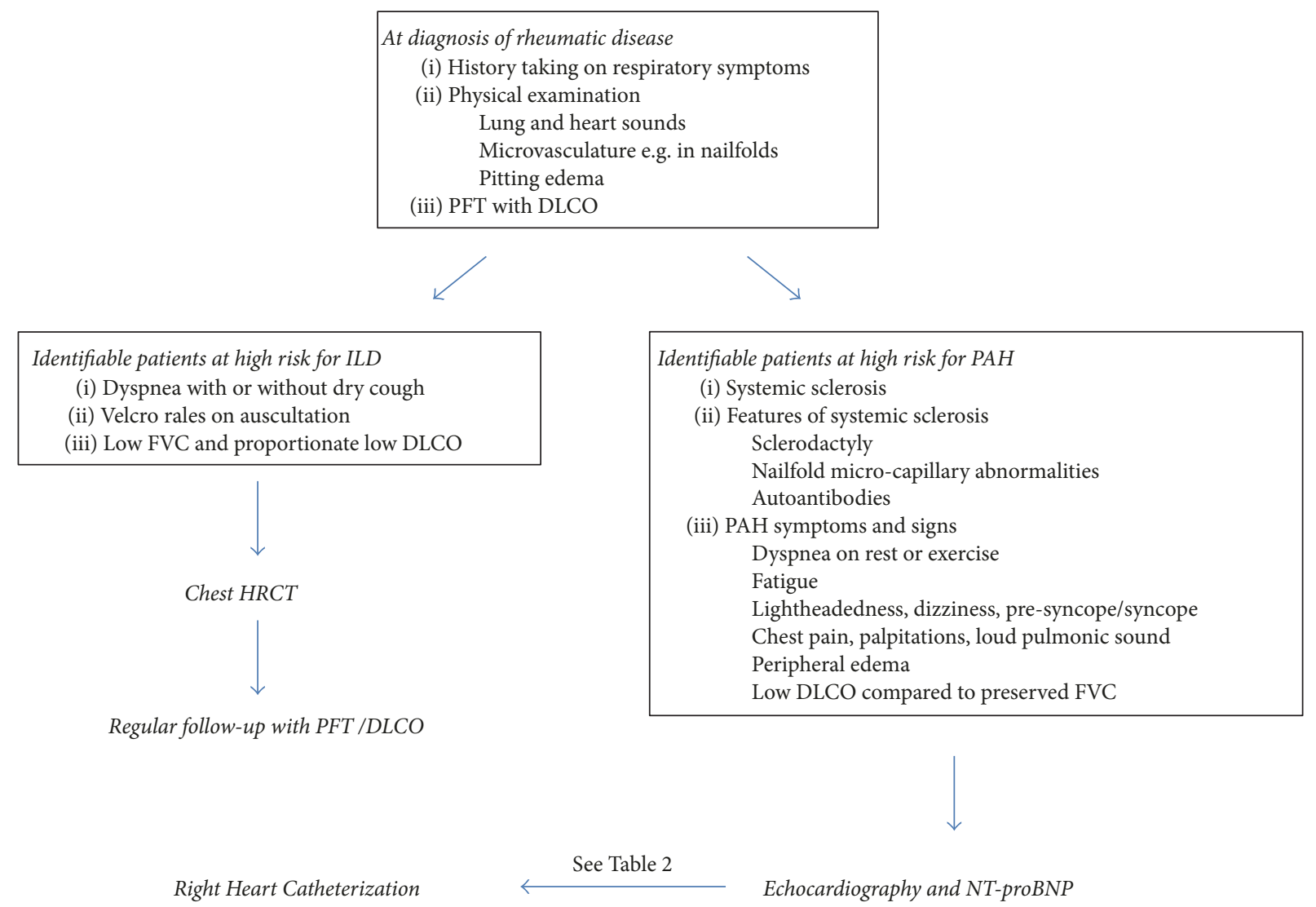

FIGURE 3: A schematic picture on screening and monitoring rheumatic disease associated lung involvements. DLCO: diffusing capacity of carbon monoxide; FVC: forced vital capacity; HRTC: high resolution computed tomography; ILD: interstitial lung disease; NT-proBNP: $\mathrm{N}$-terminal probrain natriuretic peptide; PAH: pulmonary arterial hypertension; TR: tricuspid regurgitation.

with the above modalities, PAH diagnosis should be made by RHC. Regarding airway diseases, their impact on the prognosis lacks data except for $\mathrm{BE}$ and there have been no recommendations of work-up or follow-up for this type of involvement.

\section{Conclusion}

Each rheumatic disease exhibits a unique pattern of lung involvements in terms of the affected lung structure and corresponding prevalence and incidence, severity, and treatment response. Although lung involvements, particularly ILD and $\mathrm{PAH}$, are associated with significant morbidity and mortality in rheumatic diseases, advanced screening modalities enable us to detect and treat them early. However, considerably variable course of lung diseases from inactive state to fatal progression even among those within the same histologic group and underlying rheumatic disease makes it difficult to establish a uniform treatment strategy. To meet this need, it is of importance to understand the epidemiological characteristics associated with lung involvements of a given rheumatic disease and to identify high risk patients who bear clinical equipoise between potentially toxic treatments and disease progression. Furthermore, understanding the different genetics working in each lung involvement of a specific rheumatic disease will help us develop targeted therapies stemming from the underlying pathogenesis.

\section{Conflicts of Interest}

The authors declare no conflicts of interest.

\section{References}

[1] A. Fischer and R. du Bois, "Interstitial lung disease in connective tissue disorders," The Lancet, vol. 380, no. 9842, pp. 689698, 2012.

[2] W. D. Travis, U. Costabel, D. M. Hansell et al., "An official American Thoracic Society/European Respiratory Society statement: Update of the international multidisciplinary classification of the idiopathic interstitial pneumonias," American Journal of Respiratory and Critical Care Medicine, vol. 188, no. 6, pp. 733748, 2013.

[3] P. A. Hodnett and D. P. Naidich, "Fibrosing interstitial lung disease: A practical high-resolution computed tomographybased approach to diagnosis and management and a review of the literature," American Journal of Respiratory and Critical Care Medicine, vol. 188, no. 2, pp. 141-149, 2013.

[4] D. Bouros, A. U. Wells, A. G. Nicholson et al., "Histopathologic subsets of fibrosing alveolitis in patients with systemic sclerosis 
and their relationship to outcome," American Journal of Respiratory and Critical Care Medicine, vol. 165, no. 12, pp. 1581-1586, 2002.

[5] H.-K. Lee, D. S. Kim, B. Yoo et al., "Histopathologic pattern and clinical features of rheumatoid arthritis-associated interstitial lung disease," CHEST, vol. 127, no. 6, pp. 2019-2027, 2005.

[6] W. W. Douglas, H. D. Tazelaar, T. E. Hartman et al., "Polymyositis-dermatomyositis-associated interstitial lung disease," American Journal of Respiratory and Critical Care Medicine, vol. 164, no. 7, pp. 1182-1185, 2001.

[7] H. P. Joo, S. K. Dong, I.-N. Park et al., "Prognosis of fibrotic interstitial pneumonia: Idiopathic versus collagen vascular disease-related subtypes," American Journal of Respiratory and Critical Care Medicine, vol. 175, no. 7, pp. 705-711, 2007.

[8] Y. Nakamura, K. Chida, T. Suda et al., "Nonspecific interstitial pneumonia in collagen vascular diseases: Comparison of the clinical characteristics and prognostic significance with usual interstitial pneumonia," Sarcoidosis Vasculitis and Diffuse Lung Diseses, vol. 20, no. 3, pp. 235-241, 2003.

[9] A. Bilgici, H. Ulusoy, O. Kuru, Ç. Çelenk, M. Ünsal, and M. Danaci, "Pulmonary involvement in rheumatoid arthritis," Rheumatology International, vol. 25, no. 6, pp. 429-435, 2005.

[10] B. Cortet, R.-M. Flipo, M. Remy-Jardin et al., "Use of high resolution computed tomography of the lungs in patients with rheumatoid arthritis," Annals of the Rheumatic Diseases, vol. 54, no. 10, pp. 815-819, 1995.

[11] S. Hassen Zrour, M. Touzi, I. Bejia et al., "Correlations between high-resolution computed tomography of the chest and clinical function in patients with rheumatoid arthritis: Prospective study in 75 patients," Joint Bone Spine, vol. 72, no. 1, pp. 41-47, 2005.

[12] G. Koduri, S. Norton, A. Young et al., "Interstitial lung disease has a poor prognosis in rheumatoid arthritis: results from an inception cohort," Rheumatology, vol. 49, no. 8, pp. 1483-1489, 2010.

[13] Z. M. Metafratzi, A. N. Georgiadis, C. V. Ioannidou et al., "Pulmonary involvement in patients with early rheumatoid arthritis," Scandinavian Journal of Rheumatology, vol. 36, no. 5, pp. 338-344, 2007.

[14] M. K. Demoruelle, M. H. Weisman, P. L. Simonian et al., "Airways abnormalities and rheumatoid arthritis-related autoantibodies in subjects without arthritis: Early injury or initiating site of autoimmunity?" Arthritis \& Rheumatology, vol. 64, no. 6, pp. 1756-1761, 2012.

[15] T. Bongartz, C. Nannini, Y. F. Medina-Velasquez et al., "Incidence and mortality of interstitial lung disease in rheumatoid arthritis: a population-based study," Arthritis \& Rheumatism, vol. 62, no. 6, pp. 1583-1591, 2010.

[16] J. Allain, A. Saraux, C. Guedes et al., "Prevalence of symptomatic bronchiectasis in patients with rheumatoid arthritis," Rev Rhum Engl Ed, vol. 64, no. 10, pp. 531-537, 1997.

[17] A. L. Olson, J. J. Swigris, D. B. Sprunger et al., "Rheumatoid arthritis-interstitial lung disease-associated mortality," American Journal of Respiratory and Critical Care Medicine, vol. 183, no. 3, pp. 372-378, 2011.

[18] E. Gabbay, R. Tarala, R. Will et al., "Interstitial lung disease in recent onset rheumatoid arthritis," American Journal of Respiratory and Critical Care Medicine, vol. 156, no. 2, pp. 528535, 1997.
[19] C. Hyldgaard, O. Hilberg, A. B. Pedersen et al., "A populationbased cohort study of rheumatoid arthritis-associated interstitial lung disease: Comorbidity and mortality," Annals of the Rheumatic Diseases, 2017.

[20] J. F. Restrepo, I. del Rincón, D. F. Battafarano, R. W. Haas, M. Doria, and A. Escalante, "Clinical and laboratory factors associated with interstitial lung disease in rheumatoid arthritis," Clinical Rheumatology, vol. 34, no. 9, pp. 1529-1536, 2015.

[21] A. D. Rocha-Muñoz, M. Ponce-Guarneros, J. I. Gamez-Nava et al., "Anti-Cyclic Citrullinated Peptide Antibodies and Severity of Interstitial Lung Disease in Women with Rheumatoid Arthritis," Journal of Immunology Research, vol. 2015, Article ID 151626, 2015.

[22] C. Johnson, "Recent advances in the pathogenesis, prediction, and management of rheumatoid arthritis-associated interstitial lung disease," Current Opinion in Rheumatology, vol. 29, no. 3, pp. 254-259, 2017.

[23] C. A. Kelly, V. Saravanan, and M. Nisar, "Rheumatoid arthritisrelated interstitial lung disease: associations, prognostic factors and physiological and radiological characteristics-a large multicentre UK study," Rheumatology, vol. 53, no. 9, pp. 1676-1682, 2014.

[24] E. J. Kim, H. R. Collard, and T. E. King Jr., "Rheumatoid arthritis-associated interstitial lung disease: The relevance of histopathologic and radiographic pattern," CHEST, vol. 136, no. 5, pp. 1397-1405, 2009.

[25] J. J. Solomon, J. H. Chung, G. P. Cosgrove et al., "Predictors of mortality in rheumatoid arthritis-associated interstitial lung disease," European Respiratory Journal, vol. 47, no. 2, pp. 588596, 2016.

[26] T. Suda, Y. Kaida, Y. Nakamura et al., "Acute exacerbation of interstitial pneumonia associated with collagen vascular diseases," Respiratory Medicine, vol. 103, no. 6, pp. 846-853, 2009.

[27] H. Hozumi, Y. Nakamura, T. Johkoh et al., "Acute exacerbation in rheumatoid arthritis-associated interstitial lung disease: A retrospective case control study," BMJ Open, vol. 3, no. 9, Article ID e003132, 2013.

[28] E. J. Kim, B. M. Elicker, F. Maldonado et al., "Usual interstitial pneumonia in rheumatoid arthritis-associated interstitial lung disease," European Respiratory Journal, vol. 35, no. 6, pp. 1322$1328,2010$.

[29] H. M. Nurmi, M. K. Purokivi, M. S. Kärkkäinen, H.-P. Kettunen, T. A. Selander, and R. L. Kaarteenaho, "Variable course of disease of rheumatoid arthritis-associated usual interstitial pneumonia compared to other subtypes," BMC Pulmonary Medicine, vol. 16, no. 1, article no. 107, p. 1, 2016.

[30] J. A. Hill, S. Southwood, A. Sette, A. M. Jevnikar, D. A. Bell, and E. Cairns, "Cutting edge: The conversion of arginine to citrulline allows for a high-affinity peptide interaction with the rheumatoid arthritis-associated HLA-DRB1 ${ }^{\star} 0401 \mathrm{MHC}$ class II molecule," The Journal of Immunology, vol. 171, no. 2, pp. 538541, 2003.

[31] A. H. M. Van Der Helm-Van Mil, K. N. Verpoort, F. C. Breedveld, T. W. J. Huizinga, R. E. M. Toes, and R. R. P. De Vries, "The HLA-DRB1 shared epitope alleles are primarily a risk factor for anti-cyclic citrullinated peptide antibodies and are not an independent risk factor for development of rheumatoid arthritis," Arthritis \& Rheumatology, vol. 54, no. 4, pp. 1117-1121, 2006.

[32] D. Makrygiannakis, M. Hermansson, A.-K. Ulfgren et al., "Smoking increases peptidylarginine deiminase 2 enzyme 
expression in human lungs and increases citrullination in BAL cells," Annals of the Rheumatic Diseases, vol. 67, no. 10, pp. 14881492, 2008.

[33] L. Klareskog, P. Stolt, K. Lundberg et al., "A new model for an etiology of rheumatoid arthritis: smoking may trigger HLA-DR (shared epitope)-restricted immune reactions to autoantigens modified by citrullination," Arthritis \& Rheumatism, vol. 54, no. 1, pp. 38-46, 2006.

[34] G. Reynisdottir, R. Karimi, V. Joshua et al., "Structural changes and antibody enrichment in the lungs are early features of anticitrullinated protein antibody-positive rheumatoid arthritis," Arthritis \& Rheumatology, vol. 66, no. 1, pp. 31-39, 2014.

[35] V. C. Willis, M. K. Demoruelle, L. A. Derber et al., "Sputum autoantibodies in patients with established rheumatoid arthritis and subjects at risk of future clinically apparent disease," Arthritis \& Rheumatology, vol. 65, no. 10, pp. 2545-2554, 2013.

[36] A. Fischer, J. J. Solomon, R. M. Du Bois et al., "Lung disease with anti-CCP antibodies but not rheumatoid arthritis or connective tissue disease," Respiratory Medicine, vol. 106, no. 7, pp. 10401047, 2012.

[37] A. J. Ytterberg, V. Joshua, G. Reynisdottir et al., "Shared immunological targets in the lungs and joints of patients with rheumatoid arthritis: Identification and validation," Annals of the Rheumatic Diseases, vol. 74, no. 9, pp. 1772-1777, 2015.

[38] J. T. Giles, S. K. Danoff, J. Sokolove et al., "Association of fine specificity and repertoire expansion of anticitrullinated peptide antibodies with rheumatoid arthritis associated interstitial lung disease," Annals of the Rheumatic Diseases, vol. 73, no. 8, pp. 1487-1494, 2014.

[39] H. Furukawa, S. Oka, K. Shimada et al., "Association of Human Leukocyte Antigen with Interstitial Lung Disease in Rheumatoid Arthritis: A protective role for shared epitope," PLoS ONE, vol. 7, no. 5, Article ID e33133, 2012.

[40] K. Migita, T. Nakamura, T. Koga, and K. Eguchi, "HLA-DRB1 alleles and rheumatoid arthritis-related pulmonary fibrosis," The Journal of Rheumatology, vol. 37, no. 1, pp. 205-207, 2010.

[41] S. Oka, H. Furukawa, K. Shimada et al., "Association of human leukocyte antigen alleles with chronic lung diseases in rheumatoid arthritis," Rheumatology, vol. 55, no. 7, Article ID kew025, pp. 1301-1307, 2016.

[42] H. Ennis, A. Gupta, J. Dawson, M. Lunt, W. Thomson, and A. Herrick, "HLA-DRB1 associations with rheumatoid arthritisrelated pulmonary fibrosis," Scandinavian Journal of Rheumatology, vol. 43, no. 1, pp. 75-76, 2014.

[43] S. T. Song, S. S. Kim, J. Y. Kim et al., "Association of Single Nucleotide Polymorphisms of PADI4 and HLA-DRB1 Alleles with Susceptibility to Rheumatoid Arthritis-Related Lung Diseases," Lung, vol. 194, no. 5, pp. 745-753, 2016.

[44] P. Juge, R. Borie, C. Kannengiesser et al., "Shared genetic predisposition in rheumatoid arthritis-interstitial lung disease and familial pulmonary fibrosis," European Respiratory Journal, vol. 49, no. 5, 2017.

[45] A. Fischer, K. K. Brown, R. M. Du Bois et al., "Mycophenolate mofetil improves lung function in connective tissue diseaseassociated interstitial lung disease," The Journal of Rheumatology, vol. 40, no. 5, pp. 640-646, 2013.

[46] G. J. Keir, T. M. Maher, D. Ming et al., "Rituximab in severe, treatment-refractory interstitial lung disease," Respirology, vol. 19, no. 3, pp. 353-359, 2014.

[47] S. Chartrand, J. J. Swigris, L. Peykova, and A. Fischer, "Rituximab for the treatment of connective tissue diseaseassociated interstitial lung disease," Sarcoidosis Vasculitis and Diffuse Lung Diseses, vol. 32, no. 4, pp. 296-304, 2016.

[48] B. Charlin, L. Brazeau-Lamontagne, R.-Y. Levesque, and A. Lussier, "Cricoarytenoiditis in rheumatoid arthritis: Comparison of fibrolaryngoscopic and high resolution computerized tomographic findings," Journal of Otolaryngology, vol. 14, no. 6, pp. 381-386, 1985.

[49] H. Hayakawa, A. Sato, S. Imokawa, M. Toyoshima, K. Chida, and M. Iwata, "Bronchiolar disease in rheumatoid arthritis," American Journal of Respiratory and Critical Care Medicine, vol. 154, no. 5, pp. 1531-1536, 1996.

[50] M. M. Wilczynska, A. M. Condliffe, and D. J. McKeon, "Coexistence of bronchiectasis and rheumatoid arthritis: revisited," Respiratory Care, vol. 58, no. 4, pp. 694-701, 2013.

[51] J. D. Chalmers and A. T. Hill, "Mechanisms of immune dysfunction and bacterial persistence in non-cystic fibrosis bronchiectasis," Molecular Immunology, vol. 55, no. 1, pp. 27-34, 2013.

[52] M. J. McMahon, D. R. Swinson, S. Shettar et al., "Bronchiectasis and rheumatoid arthritis: A clinical study," Annals of the Rheumatic Diseases, vol. 52, no. 11, pp. 776-779, 1993.

[53] N. A. Shadick, C. H. Fanta, M. E. Weinblatt, W. O'donnell, and J. S. Coblyn, "Bronchiectasis: A late feature of severe rheumatoid arthritis," Medicine (United States), vol. 73, no. 3, pp. 161-170, 1994.

[54] M. Wilsher, L. Voight, D. Milne et al., "Prevalence of airway and parenchymal abnormalities in newly diagnosed rheumatoid arthritis," Respiratory Medicine, vol. 106, no. 10, pp. 1441-1446, 2012.

[55] E. Perry, P. Eggleton, A. De Soyza, D. Hutchinson, and C. Kelly, "Increased disease activity, severity and autoantibody positivity in rheumatoid arthritis patients with co-existent bronchiectasis," International Journal of Rheumatic Diseases, 2015.

[56] D. R. Swinson, D. Symmons, U. Suresh, M. Jones, and J. Booth, "Decreased survival in patients with co-existent rheumatoid arthritis and bronchiectasis," British Journal of Rheumatology, vol. 36, no. 6, pp. 689-691, 1997.

[57] A.-M. Quirke, E. Perry, A. Cartwright et al., "Bronchiectasis is a model for chronic bacterial infection inducing autoimmunity in rheumatoid arthritis," Arthritis \& Rheumatology, vol. 67, no. 9, pp. 2335-2342, 2015.

[58] V. V. Kaushik, D. Hutchinson, J. Desmond, M. P. Lynch, and J. K. Dawson, "Association between bronchiectasis and smoking in patients with rheumatoid arthritis," Annals of the Rheumatic Diseases, vol. 63, no. 8, pp. 1001-1002, 2004.

[59] W. U. Hassan, N. P. Keaney, C. D. Holland, and C. A. Kelly, "High resolution computed tomography of the lung in lifelong non-smoking patients with rheumatoid arthritis," Annals of the Rheumatic Diseases, vol. 54, no. 4, pp. 308-310, 1995.

[60] S. Mori, Y. Koga, and M. Sugimoto, "Different risk factors between interstitial lung disease and airway disease in rheumatoid arthritis," Respiratory Medicine, vol. 106, no. 11, pp. 15911599, 2012.

[61] M. C. Hillarby, M. J. Mcmahon, D. M. Grennan et al., "Hla associations in subjects with rheumatoid arthritis and bronchiectasis but not with other pulmonary complications of rheumatoid disease," Rheumatology, vol. 32, no. 9, pp. 794-797, 1993.

[62] X. Puéchal, I. Fajac, T. Bienvenu et al., "Increased frequency of cystic fibrosis $\Delta$ F508 mutation in bronchiectasis associated with 
rheumatoid arthritis," European Respiratory Journal, vol. 13, no. 6, pp. 1281-1287, 1999.

[63] D. Tansey, A. U. Wells, T. V. Colby et al., "Variations in histological patterns of interstitial pneumonia between connective tissue disorders and their relationship to prognosis," Histopathology, vol. 44, no. 6, pp. 585-596, 2004.

[64] G. Devouassoux, V. Cottin, H. Liote et al., "Characterisation of severe obliterative bronchiolitis in rheumatoid arthritis," European Respiratory Journal, vol. 33, no. 5, pp. 1053-1061, 2009.

[65] A. Balbir-Gurman, M. Yigla, A. M. Nahir, and Y. BraunMoscovici, "Rheumatoid Pleural Effusion," Seminars in Arthritis and Rheumatism, vol. 35, no. 6, pp. 368-378, 2006.

[66] V. D. Steen and T. A. Medsger, "Changes in causes of death in systemic sclerosis, 1972-2002," Annals of the Rheumatic Diseases, vol. 66, no. 7, pp. 940-944, 2007.

[67] Y. Hamaguchi, "Autoantibody profiles in systemic sclerosis: predictive value for clinical evaluation and prognosis," The Journal of Dermatology, vol. 37, no. 1, pp. 42-53, 2010.

[68] H. Schurawitzki, R. Stiglbauer, W. Graninger et al., "Interstitial lung disease in progressive systemic sclerosis: High-resolution CT versus radiography," Radiology, vol. 176, no. 3, pp. 755-759, 1990.

[69] Y. A. Suliman, R. Dobrota, D. Huscher et al., "Pulmonary function tests: High rate of false-negative results in the early detection and screening of scleroderma-related interstitial lung disease," Arthritis \& Rheumatology, vol. 67, no. 12, pp. 32563261, 2015.

[70] U. A. Walker, A. Tyndall, and L. Czirjak, "Clinical risk assessment of organ manifestations in systemic sclerosis: a report from the EULAR scleroderma trails and research give up database," Annals of the Rheumatic Diseases, vol. 66, no. 6, pp. 754-763, 2007.

[71] V. D. Steen, C. Conte, G. R. Owens, and T. A. Medsger, "Severe restrictive lung disease in systemic sclerosis," Arthritis \& Rheumatism, vol. 37, no. 9, pp. 1283-1289, 1994.

[72] A. Man, T. Davidyock, L. T. Ferguson, M. Ieong, Y. Zhang, and R. W. Simms, "Changes in forced vital capacity over time in systemic sclerosis: Application of group-based trajectory modelling," Rheumatology, vol. 54, no. 8, Article ID kev016, pp. 1464-1471, 2015.

[73] S. C. Plastiras, S. P. Karadimitrakis, P. D. Ziakas, P. G. Vlachoyiannopoulos, H. M. Moutsopoulos, and G. E. Tzelepis, "Scleroderma lung: Initial forced vital capacity as predictor of pulmonary function decline," Arthritis Care \& Research, vol. 55, no. 4, pp. 598-602, 2006.

[74] D. Khanna, C.-H. Tseng, N. Farmani et al., "Clinical course of lung physiology in patients with scleroderma and interstitial lung disease: analysis of the Scleroderma Lung Study Placebo Group," Arthritis \& Rheumatology, vol. 63, no. 10, pp. 30783085, 2011.

[75] A.-M. Hoffmann-Vold, T. M. Aaløkken, M. B. Lund et al., "Predictive value of serial high-resolution computed tomography analyses and concurrent lung function tests in systemic sclerosis," Arthritis \& Rheumatology, vol. 67, no. 8, pp. 22052212, 2015.

[76] N. S. L. Goh, S. R. Desai, S. Veeraraghavan et al., "Interstitial lung disease in systemic sclerosis: a simple staging system," American Journal of Respiratory and Critical Care Medicine, vol. 177, no. 11, pp. 1248-1254, 2008.

[77] I. M. Markusse, J. Meijs, B. de Boer et al., "Predicting cardiopulmonary involvement in patients with systemic sclerosis:
Complementary value of nailfold videocapillaroscopy patterns and disease-specific autoantibodies," Rheumatology, vol. 56, no. 7, pp. 1081-1088, 2017.

[78] S. Assassi, R. Sharif, R. E. Lasky et al., "Predictors of interstitial lung disease in early systemic sclerosis: A prospective longitudinal study of the GENISOS cohort," Arthritis Research \& Therapy, p. R166, 2010.

[79] M. Peters-Golden, R. A. Wise, P. Schneider, M. Hochberg, M. B. Stevens, and F. Wigley, "Clinical and demographic predictors of loss of pulmonary function in systemic sclerosis," Medicine (United States), vol. 63, no. 4, pp. 221-231, 1984.

[80] C. Richardson, R. Agrawal, J. Lee et al., "Esophageal dilatation and interstitial lung disease in systemic sclerosis: A crosssectional study," Seminars in Arthritis and Rheumatism, vol. 46, no. 1, pp. 109-114, 2016.

[81] I. Marie, S. Dominique, H. Levesque et al., "Esophageal involvement and pulmonary manifestations in systemic sclerosis," Arthritis Care \& Research, vol. 45, no. 4, pp. 346-354, 2001.

[82] X. J. Zhang, A. Bonner, M. Hudson, M. Baron, and J. Pope, "Association of gastroesophageal factors and worsening of forced vital capacity in systemic sclerosis," The Journal of Rheumatology, vol. 40, no. 6, pp. 850-858, 2013.

[83] R. B. C. De Souza, C. T. L. Borges, V. L. Capelozzi et al., "Centrilobular fibrosis: An underrecognized pattern in systemic sclerosis," Respiration, vol. 77, no. 4, pp. 389-397, 2009.

[84] G. Lepri, S. Guiducci, S. Bellando-Randone et al., "Evidence for oesophageal and anorectal involvement in very early systemic sclerosis (VEDOSS): Report from a single VEDOSS/EUSTAR centre," Annals of the Rheumatic Diseases, vol. 74, no. 1, pp. 124128, 2015.

[85] K. A. Johannson, I. Strambu, and C. Ravaglia, "Antacid therapy in idiopathic pulmonary fibrosis: more questions than answers?" The Lancet Respiratory Medicine, vol. 5, no. 7, pp. 591598, 2017.

[86] M. Kreuter, W. Wuyts, E. Renzoni et al., "Antacid therapy and disease outcomes in idiopathic pulmonary fibrosis: A pooled analysis," The Lancet Respiratory Medicine, vol. 4, no. 5, pp. 381389, 2016.

[87] J. S. Lee, H. R. Collard, K. J. Anstrom et al., "Anti-acid treatment and disease progression in idiopathic pulmonary fibrosis: an analysis of data from three randomised controlled trials," The Lancet Respiratory Medicine, vol. 1, no. 5, pp. 369-376, 2013.

[88] R. M. Shah, S. Jimenez, and R. Wechsler, "Significance of ground-glass opacity on HRCT in long-term follow-up of patients with systemic sclerosis," Journal of Thoracic Imaging, vol. 22, no. 2, pp. 120-124, 2007.

[89] D. Launay, M. Remy-Jardin, U. Michon-Pasturel et al., "High resolution computed tomography in fibrosing alveolitis associated with systemic sclerosis," The Journal of Rheumatology, vol. 33, no. 9, pp. 1789-1801, 2006.

[90] J. W. Song, K.-H. Do, M.-Y. Kim, S. J. Jang, T. V. Colby, and D. S. Kim, "Pathologic and radiologic differences between idiopathic and collagen vascular disease-related usual interstitial pneumonia," CHEST, vol. 136, no. 1, pp. 23-30, 2009.

[91] M. Rubio-Rivas, C. Royo, C. P. Simeón, X. Corbella, and V. Fonollosa, "Mortality and survival in systemic sclerosis: Systematic review and meta-analysis," Seminars in Arthritis and Rheumatism, vol. 44, no. 2, pp. 208-219, 2015.

[92] T. R. Radstake, O. Gorlova, B. Rueda et al., "Genome-wide association study of systemic sclerosis identifies CD247 as a new susceptibility locus," Nature Genetics, vol. 42, no. 5, pp. 426-429, 2010. 
[93] P. S. Ramos, R. M. Silver, and C. A. Feghali-Bostwick, "Genetics of systemic sclerosis: Recent advances," Current Opinion in Rheumatology, vol. 27, no. 6, pp. 521-529, 2015.

[94] L. Bossini-Castillo, E. López-Isac, M. D. Mayes, and J. Martín, "Genetics of systemic sclerosis," Seminars in Immunopathology, vol. 37, no. 5, pp. 443-451, 2015.

[95] D. P. Tashkin, R. Elashoff, P. J. Clements et al., "Cyclophosphamide versus placebo in scleroderma lung disease," The New England Journal of Medicine, vol. 354, no. 25, pp. 2655-2666, 2006.

[96] D. P. Tashkin, M. D. Roth, and P. J. Clements, "Mycophenolate mofetil versus oral cyclophosphamide in scleroderma-related interstitial lung disease (SLS II): a randomised controlled, double-blind, parallel group trial," The Lancet Respiratory Medicine, vol. 4, no. 9, pp. 708-719, 2016.

[97] C. Fonseca, G. E. Lindahl, M. Ponticos et al., "A polymorphism in the CTGF promoter region associated with systemic sclerosis," The New England Journal of Medicine, vol. 357, no. 12, pp. 1210-1220, 2007.

[98] Y. Kawaguchi, Y. Ota, M. Kawamoto et al., "Association study of a polymorphism of the CTGF gene and susceptibility to systemic sclerosis in the Japanese population," Annals of the Rheumatic Diseases, vol. 68, no. 12, pp. 1921-1924, 2009.

[99] A. C. Y. Mak, P. L. F. Tang, C. Cleveland et al., "Brief Report: Whole-Exome Sequencing for Identification of Potential Causal Variants for Diffuse Cutaneous Systemic Sclerosis," Arthritis \& Rheumatology, vol. 68, no. 9, pp. 2257-2262, 2016.

[100] M. Ciechomska, J. M. Van Laar, and S. O’Reilly, "Emerging role of epigenetics in systemic sclerosis pathogenesis," Genes \& Immunity, vol. 15, no. 7, pp. 433-439, 2014.

[101] J. C. A. Broen, T. R. D. J. Radstake, and M. Rossato, "The role of genetics and epigenetics in the pathogenesis of systemic sclerosis," Nature Reviews Rheumatology, vol. 10, no. 11, pp. 671681, 2014.

[102] P. Dieudé, M. Guedj, J. Wipff et al., "Association between the IRF5 rs2004640 functional polymorphism and systemic sclerosis: A new perspective for pulmonary fibrosis," Arthritis \& Rheumatology, vol. 60, no. 1, pp. 225-233, 2009.

[103] R. Sharif, M. D. Mayes, F. K. Tan et al., "IRF5 polymorphism predicts prognosis in patients with systemic sclerosis," Annals of the Rheumatic Diseases, vol. 71, no. 7, pp. 1197-1202, 2012.

[104] P. Dieudé, M. Guedj, J. Wipff et al., "STAT4 is a genetic risk factor for systemic sclerosis having additive effects with IRF5 on disease susceptibility and related pulmonary fibrosis," Arthritis \& Rheumatology, vol. 60, no. 8, pp. 2472-2479, 2009.

[105] O. Kowal-Bielecka, S. Chwiesko-Minarowska, P. L. Bernatowicz et al., "The arachidonate 5-lipoxygenase activating protein gene polymorphism is associated with the risk of sclerodermarelated interstitial lung disease: A multicentre European Scleroderma Trials and Research group (EUSTAR) study," Rheumatology, vol. 56, no. 5, pp. 844-852, 2017.

[106] P. Dieudé, M. Guedj, J. Wipff et al., "NLRP1 influences the systemic sclerosis phenotype: A new clue for the contribution of innate immunity in systemic sclerosis-related fibrosing alveolitis pathogenesis," Annals of the Rheumatic Diseases, vol. 70, no. 4, pp. 668-674, 2011.

[107] L. Bossini-Castillo, C. P. Simeon, L. Beretta et al., "A multicenter study confirms CD226 gene association with systemic sclerosisrelated pulmonary fibrosis," Arthritis Research \& Therapy, vol. 14, no. 2, article no. R85, 2012.

[108] K. Hoshino, T. Satoh, Y. Kawaguchi, and M. Kuwana, "Association of hepatocyte growth factor promoter polymorphism with severity of interstitial lung disease in Japanese patients with systemic sclerosis," Arthritis \& Rheumatology, vol. 63, no. 8, pp. 2465-2472, 2011.

[109] M. Wu, S. Assassi, G. A. Salazar et al., "Genetic susceptibility loci of idiopathic interstitial pneumonia do not represent risk for systemic sclerosis: A case control study in Caucasian patients," Arthritis Research \& Therapy, vol. 18, no. 1, article no. 20, 2016.

[110] A. L. Peljto, M. P. Steele, T. E. Fingerlin et al., "The pulmonary fibrosis-associated MUC5B promoter polymorphism does not influence the development of interstitial pneumonia in systemic sclerosis," CHEST, vol. 142, no. 6, pp. 1584-1588, 2012.

[111] R. Borie, B. Crestani, P. Dieude et al., "The MUC5B variant is associated with idiopathic pulmonary fibrosis but not with systemic sclerosis interstitial lung disease in the european caucasian population," PLoS ONE, vol. 8, no. 8, Article ID e70621, 2013.

[112] C. J. Stock, H. Sato, C. Fonseca et al., "Mucin 5B promoter polymorphism is associated with idiopathic pulmonary fibrosis but not with development of lung fibrosis in systemic sclerosis or sarcoidosis," Thorax, vol. 68, no. 5, pp. 436-441, 2013.

[113] D. P. Tashkin, R. Elashoff, P. J. Clements et al., "Effects of 1-year treatment with cyclophosphamide on outcomes at 2 years in scleroderma lung disease," American Journal of Respiratory and Critical Care Medicine, vol. 176, no. 10, pp. 1026-1034, 2007.

[114] R. K. Hoyles, R. W. Ellis, J. Wellsbury et al., "A multicenter, prospective, randomized, double-blind, placebo-controlled trial of corticosteroids and intravenous cyclophosphamide followed by oral azathioprine for the treatment of pulmonary fibrosis in scleroderma," Arthritis \& Rheumatology, vol. 54, no. 12, pp. 3962-3970, 2006.

[115] D. Khanna, C. Denton, C. J. Lin et al., "Safety and efficacy of subcutaneous tocilizumab in systemic sclerosis: results from the open-label period of a phase II randomised controlled trial (faSScinate)," Annals of the Rheumatic Diseases, vol. 77, no. 2, pp. 212-220, 2018.

[116] D. Daoussis, S.-N. C. Liossis, A. C. Tsamandas et al., "Experience with rituximab in scleroderma: Results from a 1-year, proof-of-principle study," Rheumatology, vol. 49, no. 2, Article ID kep093, pp. 271-280, 2010.

[117] E. Hachulla, V. Gressin, L. Guillevin et al., "Early detection of pulmonary arterial hypertension in systemic sclerosis: a French nationwide prospective multicenter study," Arthritis \& Rheumatology, vol. 52, no. 12, pp. 3792-3800, 2005.

[118] D. Mukerjee, D. St George, B. Coleiro et al., "Prevalence and outcome in systemic sclerosis associated pulmonary arterial hypertension: application of a registry approach," Annals of the Rheumatic Diseases, vol. 62, no. 11, pp. 1088-1093, 2003.

[119] R. G. Ungerer, D. P. Tashkin, D. Furst et al., "Prevalence and clinical correlates of pulmonary arterial hypertension in progressive systemic sclerosis," American Journal of Medicine, vol. 75, no. 1, pp. 65-74, 1983.

[120] E. Hachulla, D. Launay, L. Mouthon et al., "Is pulmonary arterial hypertension really a late complication of systemic sclerosis?" CHEST, vol. 136, no. 5, pp. 1211-1219, 2009.

[121] M. Humbert, A. Yaici, P. De Groote et al., "Screening for pulmonary arterial hypertension in patients with systemic sclerosis: Clinical characteristics at diagnosis and long-term survival," Arthritis \& Rheumatology, vol. 63, no. 11, pp. 35223530, 2011.

[122] J. J. Solomon, A. L. Olson, A. Fischer, T. Bull, K. K. Brown, and G. Raghu, "Scleroderma lung disease," European Respiratory Review, vol. 22, no. 127, pp. 6-19, 2013. 
[123] V. Thakkar, W. M. Stevens, D. Prior et al., "N-terminal probrain natriuretic peptide in a novel screening algorithm for pulmonary arterial hypertension in systemic sclerosis: A casecontrol study," Arthritis Research \& Therapy, vol. 14, no. 3, article no. R143, 2012.

[124] M. H. Williams, C. E. Handler, R. Akram et al., "Role of $\mathrm{N}$-terminal brain natriuretic peptide (N-TproBNP) in scleroderma-associated pulmonary arterial hypertension," European Heart Journal, vol. 27, no. 12, pp. 1485-1494, 2006.

[125] V. Steen and T. A. Medsger Jr., "Predictors of isolated pulmonary hypertension in patients with systemic sclerosis and limited cutaneous involvement," Arthritis \& Rheumatology, vol. 48, no. 2, pp. 516-522, 2003.

[126] J. G. Coghlan, C. P. Denton, E. Grünig et al., "Evidence-based detection of pulmonary arterial hypertension in systemic sclerosis: The DETECT study," Annals of the Rheumatic Diseases, vol. 73, no. 7, pp. 1340-1349, 2014.

[127] C. P. Denton, J. B. Cailes, G. D. Phillips, A. U. Wells, C. M. Black, and R. M. D. U. Bois, "Comparison of Doppler echocardiography and right heart catheterization to assess pulmonary hypertension in systemic sclerosis," British Journal of Rheumatology, vol. 36, no. 2, pp. 239-243, 1997.

[128] V. M. Hsu, A. E. Moreyra, and A. C. Wilson, "Assessment of pulmonary arterial hypertension in patients with systemic sclerosis: comparison of noninvasive tests with results of rightheart catheterization," The Journal of Rheumatology, vol. 35, no. 3, pp. 458-465, 2008.

[129] T. Soukup, R. Pudil, K. Kubinova et al., "Application of the DETECT algorithm for detection of risk of pulmonary arterial hypertension in systemic sclerosis: Data from a Czech tertiary centre," Rheumatology, vol. 55, no. 1, Article ID kev327, pp. 109114, 2016.

[130] C. Mihai, M. Antic, R. Dobrota et al., "Factors associated with disease progression in early-diagnosed pulmonary arterial hypertension associated with systemic sclerosis: longitudinal data from the DETECT cohort," Annals of the Rheumatic Diseases, vol. 77, no. 1, pp. 128-132, 2018.

[131] S. C. Mathai, L. K. Hummers, H. C. Champion et al., "Survival in pulmonary hypertension associated with the scleroderma spectrum of diseases: Impact of interstitial lung disease," Arthritis \& Rheumatology, vol. 60, no. 2, pp. 569-577, 2009.

[132] G. Lefèvre, L. Dauchet, E. Hachulla et al., "Survival and prognostic factors in systemic sclerosis-associated pulmonary hypertension: A systematic review and meta-analysis," Arthritis \& Rheumatology, vol. 65, no. 9, pp. 2412-2423, 2013.

[133] A. Komócsi, A. Vorobcsuk, R. Faludi et al., "The impact of cardiopulmonary manifestations on the mortality of SSC: A systematic review and meta-analysis of observational studies," Rheumatology, vol. 51, no. 6, Article ID ker357, pp. 1027-1036, 2012.

[134] L. Chung, J. Liu, L. Parsons et al., "Characterization of connective tissue disease-associated pulmonary arterial hypertension from REVEAL: identifying systemic sclerosis as a unique phenotype," Chest, vol. 138, no. 6, pp. 1383-1394, 2010.

[135] L. Bossini-Castillo, D. Campillo-Davo, E. Lopez-Isac et al., "An MIF promoter polymorphism is associated with susceptibility to pulmonary arterial hypertension in diffuse cutaneous systemic sclerosis," The Journal of Rheumatology, vol. 44, no. 10, pp. 1453-1457, 2017.

[136] R. G. Marangoni, B. D. Korman, Y. Allanore et al., "A candidate gene study reveals association between a variant of the Peroxisome Proliferator-Activated Receptor Gamma (PPAR- $\gamma$ ) gene and systemic sclerosis," Arthritis Research \& Therapy, vol. 17, no. 1, article no. 128, 2015.

[137] J. Wipff, P. Dieudé, M. Guedj et al., "Association of a KCNA5 gene polymorphism with systemic sclerosis-associated pulmonary arterial hypertension in the European Caucasian population," Arthritis \& Rheumatology, vol. 62, no. 10, pp. 3093-3100, 2010.

[138] L. Bossini-Castillo, C. P. Simeon, L. Beretta et al., "KCNA5 gene is not confirmed as a systemic sclerosis-related pulmonary arterial hypertension genetic susceptibility factor," Arthritis Research \& Therapy, vol. 14, no. 6, article no. R273, 2012.

[139] J. C. A. Broen, L. Bossini-Castillo, L. van Bon et al., "A rare polymorphism in the gene for Toll-like receptor 2 is associated with systemic sclerosis phenotype and increases the production of inflammatory mediators," Arthritis \& Rheumatology, vol. 64, no. 1, pp. 264-271, 2012.

[140] M. Manetti, Y. Allanore, and L. Revillod, "A genetic variation located in the promoter region of the UPAR (CD87) gene is associated with the vascular complications of systemic sclerosis," Arthritis \& Rheumatology, vol. 63, pp. 247-256, 2011.

[141] P. Dieudé, M. Guedj, J. Wipff et al., "Association of the TNFAIP3 rs5029939 variant with systemic sclerosis in the European Caucasian population," Annals of the Rheumatic Diseases, vol. 69, no. 11, pp. 1958-1964, 2010.

[142] S. K. Agarwal, P. Gourh, S. Shete et al., "Association of interleukin 23 receptor polymorphisms with anti-topoisomerase-I positivity and pulmonary hypertension in systemic sclerosis," The Journal of Rheumatology, vol. 36, no. 12, pp. 2715-2723, 2009.

[143] M. Manetti, V. Liakouli, C. Fatini et al., "Association between a stromal cell-derived factor 1 (SDF-1/CXCL12) gene polymorphism and microvascular disease in systemic sclerosis," Annals of the Rheumatic Diseases, vol. 68, no. 3, pp. 408-411, 2009.

[144] D. Khanna, H. Gladue, R. Channick et al., "Recommendations for screening and detection of connective tissue disease-associated pulmonary arterial hypertension," Arthritis \& Rheumatology, vol. 65, no. 12, pp. 3194-3201, 2013.

[145] V. McLaughlin, M. Humbert, G. Coghlan, P. Nash, and V. Steen, "Pulmonary arterial hypertension: the most devastating vascular complication of systemic sclerosis," Rheumatology, vol. 48, supplement 3, pp. iii25-31, 2009.

[146] T. Pulido, I. Adzerikho, R. N. Channick et al., "Macitentan and morbidity and mortality in pulmonary arterial hypertension," The New England Journal of Medicine, vol. 369, no. 9, pp. 809818, 2013.

[147] N. Galie, J. A. Barbera, A. E. Frost et al., "Initial use of ambrisentan plus tadalafil in pulmonary arterial hypertension," The New England Journal of Medicine, vol. 373, no. 9, pp. 834844, 2015.

[148] J. G. Coghlan, N. Galie, J. A. Barbera et al., "Initial combination therapy with ambrisentan and tadalafil in connective tissue disease-associated pulmonary arterial hypertension (CTD$\mathrm{PAH}$ ): subgroup analysis from the AMBITION trial," Annals of the Rheumatic Diseases, vol. 76, no. 7, pp. 1219-1227, 2017.

[149] O. Sitbon, R. Channick, K. M. Chin et al., "Selexipag for the treatment of pulmonary arterial hypertension," The New England Journal of Medicine, vol. 373, no. 26, pp. 2522-2533, 2015.

[150] S. Gaine, K. Chin, G. Coghlan et al., "Selexipag for the treatment of connective tissue disease-associated pulmonary arterial hypertension," European Respiratory Journal, vol. 50, no. 2, 2017. 
[151] S. Sanges, C. M. Yelnik, O. Sitbon et al., "Pulmonary arterial hypertension in idiopathic inflammatory myopathies Data from the French pulmonary hypertension registry and review of the literature," Medicine (United States), vol. 95, no. 39, 2016.

[152] I. Marie, E. Hachulla, P. Cherin et al., "Interstitial lung disease in polymyositis and dermatomyositis," Arthritis \& Rheumatism, vol. 47, no. 6, pp. 614-622, 2002.

[153] M. Fathi, M. Dastmalchi, E. Rasmussen, I. E. Lundberg, and G. Tornling, "Interstitial lung disease, a common manifestation of newly diagnosed polymyositis and dermatomyositis," Annals of the Rheumatic Diseases, vol. 63, no. 3, pp. 297-301, 2004.

[154] T. Mimori, R. Nakashima, and Y. Hosono, "Interstitial lung disease in myositis: Clinical subsets, biomarkers, and treatment," Current Rheumatology Reports, vol. 14, no. 3, pp. 264-274, 2012.

[155] V. Cottin, F. Thivolet-Béjui, M. Reynaud-Gaubert et al., "Interstitial lung disease in amyopathic dermatomyositis, dermatomyositis and polymyositis," European Respiratory Journal, vol. 22, no. 2, pp. 245-250, 2003.

[156] B. F. Dickey and A. R. Myers, "Pulmonary disease in polymyositis/dermatomyositis," Seminars in Arthritis and Rheumatism, vol. 14, no. 1, pp. 60-76, 1984.

[157] E. H. Kang, E. B. Lee, K. C. Shin et al., "Interstitial lung disease in patients with polymyositis, dermatomyositis and amyopathic dermatomyositis," Rheumatology, vol. 44, no. 10, pp. 1282-1286, 2005.

[158] R. L. Euwer and R. D. Sontheimer, "Amyopathic dermatomyositis (dermatomyositis siné myositis): Presentation of six new cases and review of the literature," Journal of the American Academy of Dermatology, vol. 24, no. 6, pp. 959-966, 1991.

[159] H. D. Tazelaar, R. W. Viggiano, J. Pickersgill, and T. V. Colby, "Interstitial lung disease in polymyositis and dermatomyositis. Clinical features and prognosis as correlated with histologic findings," American Review of Respiratory Disease, vol. 141, no. 3 I, pp. 727-733, 1990.

[160] K. Fujikawa, A. Kawakami, K. Kaji et al., "Association of distinct clinical subsets with myositisspecific autoantibodies towards anti155/140kDa polypeptides, anti-140-kDa polypeptides, and antiaminoacyl tRNA synthetases in Japanese patients with dermatomyositis: a singlecentre, crosssectional study," Scandinavian Journal of Rheumatology, vol. 38, no. 4, pp. 263-267, 2009.

[161] S. Sato, K. Hoshino, T. Satoh, T. Fujita, Y. Kawakami, and M. Kuwana, "RNA helicase encoded by melanoma differentiationassociated gene 5 is a major autoantigen in patients with clinically amyopathic dermatomyositis: association with rapidly progressive interstitial lung disease," Arthritis \& Rheumatology, vol. 60, no. 7, pp. 2193-2200, 2009.

[162] F. W. Miller, W. Chen, T. P. O’Hanlon et al., “Genome-wide association study identifies HLA 8.1 ancestral haplotype alleles as major genetic risk factors for myositis phenotypes," Genes \& Immunity, vol. 16, no. 7, pp. 470-480, 2015.

[163] F. W. Miller, R. G. Cooper, J. Vencovský et al., “Genome-wide association study of dermatomyositis reveals genetic overlap with other autoimmune disorders," Arthritis \& Rheumatology, vol. 65, no. 12, pp. 3239-3247, 2013.

[164] M. Jani, J. Massey, L. R. Wedderburn et al., "Genotyping of immune-related genetic variants identifies TYK2 as a novel associated locus for idiopathic inflammatory myopathies," Annals of the Rheumatic Diseases, vol. 73, no. 9, pp. 1750-1752, 2014.
[165] T. Sugiura, Y. Kawaguchi, K. Goto et al., "Association between a C8orf13-BLK polymorphism and polymyositis/ dermatomyositis in the Japanese population: An additive effect with STAT4 on disease susceptibility," PLoS ONE, vol. 9, no. 3, Article ID e90019, 2014.

[166] S. Chen, W. Wu, J. Li et al., "Single nucleotide polymorphisms in the FAM167A-BLK gene are associated with polymyositis/dermatomyositis in the Han Chinese population," Immunologic Research, vol. 62, no. 2, article no. 8646, pp. 153-162, 2015.

[167] S. Rothwell, R. G. Cooper, I. E. Lundberg et al., "Dense genotyping of immune-related loci in idiopathic inflammatory myopathies confirms HLA alleles as the strongest genetic risk factor and suggests different genetic background for major clinical subgroups," Annals of the Rheumatic Diseases, vol. 75, no. 8, pp. 1558-1566, 2016.

[168] T. Sugiura, Y. Kawaguchi, K. Goto et al., "Positive association between STAT4 polymorphisms, and polymyositis/ dermatomyositis in a Japanese population," Annals of the Rheumatic Diseases, vol. 71, no. 10, pp. 1646-1650, 2012.

[169] S. Chen, Q. Wang, C. Y. Wu et al., "A single-nucleotide polymorphism of CCL21 rs951005 T>C is associated with susceptibility of polymyositis and such patients with interstitial lung disease in a Chinese Han population," Clinical and Experimental Rheumatology, vol. 33, no. 5, pp. 639-646, 2015.

[170] S. Chen, Q. Wang, Z. Wu et al., "Genetic association study of TNFAIP3, IFIH1, IRF5 polymorphisms with polymyositis/dermatomyositis in Chinese Han population," PLoS ONE, vol. 9, no. 10, Article ID el10044, 2014.

[171] Q. Wang, S. Chen, Y. Li et al., "Positive association of genetic variations in the phospholipase C-like 1 gene with dermatomyositis in Chinese Han," Immunologic Research, vol. 64, no. 1, pp. 204-212, 2016.

[172] H. Andersson, M. Sem, M. B. Lund et al., "Long-term experience with rituximab in anti-synthetase syndrome-related interstitial lung disease," Rheumatology, vol. 54, no. 8, Article ID kev004, pp. 1420-1428, 2015.

[173] C. V. Oddis, "Update on the pharmacological treatment of adult myositis," Journal of Internal Medicine, vol. 280, no. 1, pp. 63-74, 2016.

[174] H. M. Fenlon, M. Doran, S. M. Sant, and É. Breatnach, "High-resolution chest CT in systemic lupus erythematosus," American Journal of Roentgenology, vol. 166, no. 2, pp. 301-307, 1996.

[175] S. M. Sant, M. Doran, H. M. Fenelon, and E. S. Breatnach, "Pleuropulmonary abnormalities in patients with systemic lupus erythematosus: Assessment with high resolution computed tomography, chest radiography and pulmonary function tests," Clinical and Experimental Rheumatology, vol. 15, no. 5, pp. 507513, 1997.

[176] A. A. Bankier, H. P. Kiener, M. N. Wiesmayr et al., "Discrete lung involvement in systemic lupus erythematosus: CT assessment," Radiology, vol. 196, no. 3, pp. 835-840, 1995.

[177] M. P. Keane and J. P. Lynch III, "Pleuropulmonary manifestations of systemic lupus erythematosus," Thorax, vol. 55, no. 2, pp. 159-166, 2000.

[178] I. C. Mira-Avendano and A. Abril, "Pulmonary manifestations of Sjögren syndrome, systemic lupus erythematosus, and mixed connective tissue disease," Rheumatic Disease Clinics of North America, vol. 41, no. 2, pp. 263-277, 2015.

[179] C. Toworakul, N. Kasitanon, W. Sukitawut, R. Wichinun, and W. Louthrenoo, "Usefulness of pleural effusion antinuclear 
antibodies in the diagnosis of lupus pleuritis," Lupus, vol. 20, no. 10, pp. 1042-1046, 2011.

[180] H. Borrell, J. Narváez, J. J. Alegre et al., "Shrinking lung syndrome in systemic lupus erythematosus: A case series and review of the literature," Medicine (United States), vol. 95, no. 33, article no. 1926, 2016.

[181] L. Duron, F. Cohen-Aubart, E. Diot et al., "Shrinking lung syndrome associated with systemic lupus erythematosus: A multicenter collaborative study of 15 new cases and a review of the 155 cases in the literature focusing on treatment response and long-term outcomes," Autoimmunity Reviews, vol. 15, no. 10, pp. 944-1000, 2016.

[182] L. A. Henderson, S. H. Loring, R. R. Gill et al., "Shrinking lung syndrome as a manifestation of pleuritis: A new model based on pulmonary physiological studies," The Journal of Rheumatology, vol. 40, no. 3, pp. 273-281, 2013.

[183] E. K. Li and L. S. Tam, "Pulmonary hypertension in systemic lupus erythematosus: clinical association and survival in 18 patients," The Journal of Rheumatology, vol. 26, no. 9, pp. 19231929, 1999.

[184] A. Prabu, K. Patel, C.-S. Yee et al., "Prevalence and risk factors for pulmonary arterial hypertension in patients with lupus," Rheumatology, vol. 48, no. 12, pp. 1506-1511, 2009.

[185] M. Li, Q. Wang, J. Zhao et al., "Chinese SLE Treatment and Research group (CSTAR) registry: II. Prevalence and risk factors of pulmonary arterial hypertension in Chinese patients with systemic lupus erythematosus," Lupus, vol. 23, no. 10, pp. 1085-1091, 2014.

[186] A. Cefle, M. Inanc, M. Sayarlioglu et al., "Pulmonary hypertension in systemic lupus erythematosus: Relationship with antiphospholipid antibodies and severe disease outcome," Rheumatology International, vol. 31, no. 2, pp. 183-189, 2011.

[187] X. Jais, D. Launay, A. Yaici et al., "Immunosuppressive therapy in lupus- and mixed connective tissue disease-associated pulmonary arterial hypertension: a retrospective analysis of twenty-three cases," Arthritis \& Rheumatology, vol. 58, no. 2, pp. 521-531, 2008.

[188] E. Tanaka, M. Harigai, M. Tanaka, Y. Kawaguchi, M. Hara, and N. Kamatani, "Pulmonary hypertension in systemic lupus erythematosus: Evaluation of clinical characteristics and response to immunosuppressive treatment," The Journal of Rheumatology, vol. 29, no. 2, pp. 282-287, 2002.

[189] S. Kommireddy, S. Bhyravavajhala, K. Kurimeti et al., "Pulmonary arterial hypertension in systemic lupus erythematosus may benefit by addition of immunosuppression to vasodilator therapy: An observational study," Rheumatology, vol. 54, no. 9, pp. 1673-1679, 2015.

[190] M. R. Zamora, M. L. Warner, R. Tuder, and M. I. Schwarz, "Diffuse alveolar hemorrhage and systemic lupus erythematosus: clinical presentation, histology, survival, and outcome," Medicine, vol. 76, no. 3, pp. 192-202, 1997.

[191] M. U. Martínez-Martínez and C. Abud-Mendoza, "Predictors of mortality in diffuse alveolar haemorrhage associated with systemic lupus erythematosus," Lupus, vol. 20, no. 6, pp. 568574, 2011.

[192] C. B. Narshi, S. Haider, C. M. Ford, D. A. Isenberg, and I. P. Giles, "Rituximab as early therapy for pulmonary haemorrhage in systemic lupus erythematosus," Rheumatology, vol. 49, no. 2, Article ID kep356, pp. 392-394, 2010.

[193] M. Gutsche, G. D. Rosen, and J. J. Swigris, "Connective tissue disease-associated interstitial lung disease: a review," Current Respiratory Care Reports, vol. 1, pp. 224-232, 2012.
[194] L. Martin, S. M. Edworthy, J. P. Ryan, and M. J. Fritzler, "Upper airway disease in systemic lupus erythematosus: A report of 4 cases and a review of the literature," The Journal of Rheumatology, vol. 19, no. 8, pp. 1186-1190, 1992. 


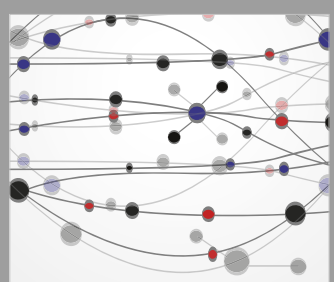

The Scientific World Journal
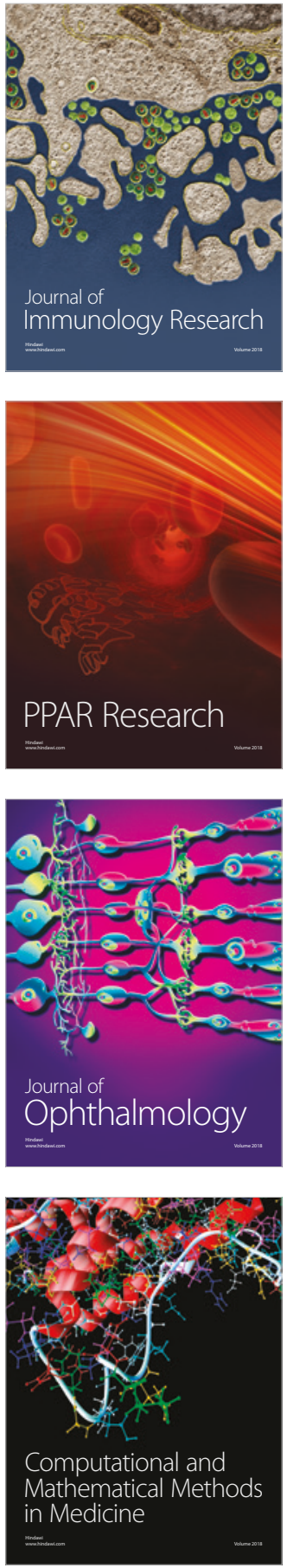

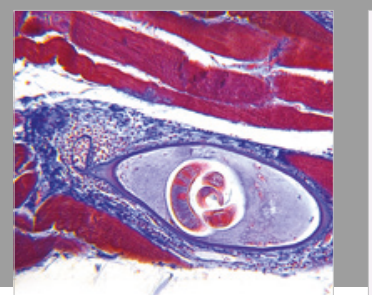

Gastroenterology Research and Practice

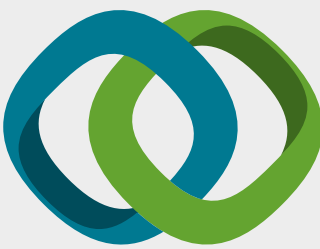

\section{Hindawi}

Submit your manuscripts at

www.hindawi.com
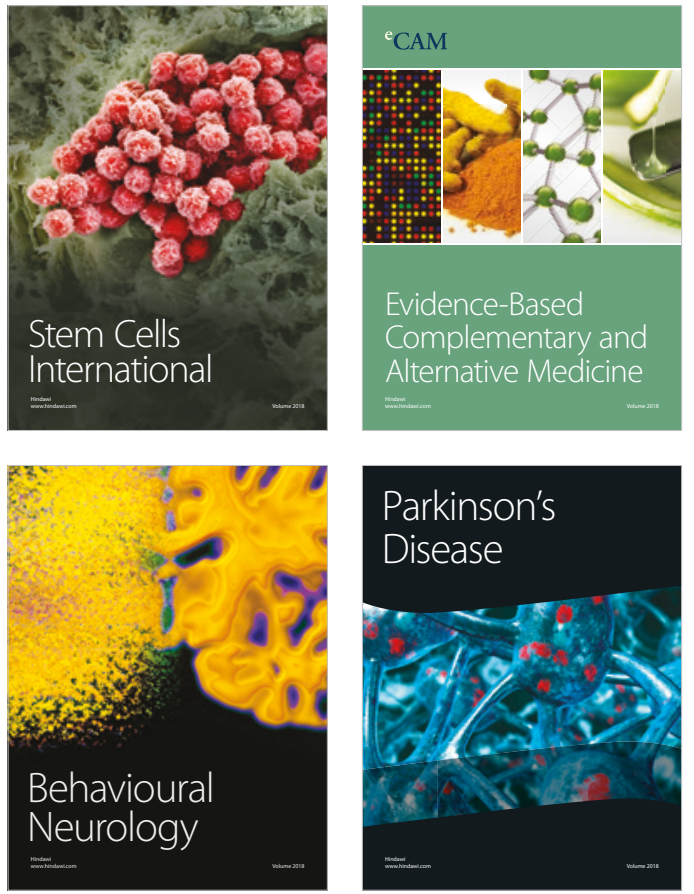

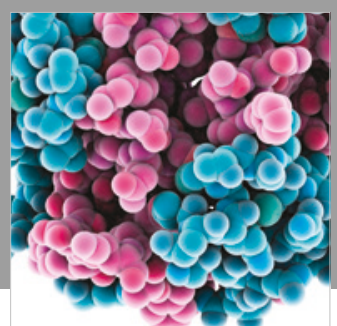

ournal of

Diabetes Research

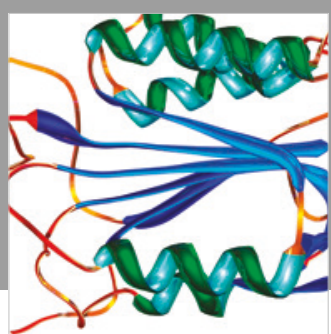

Disease Markers
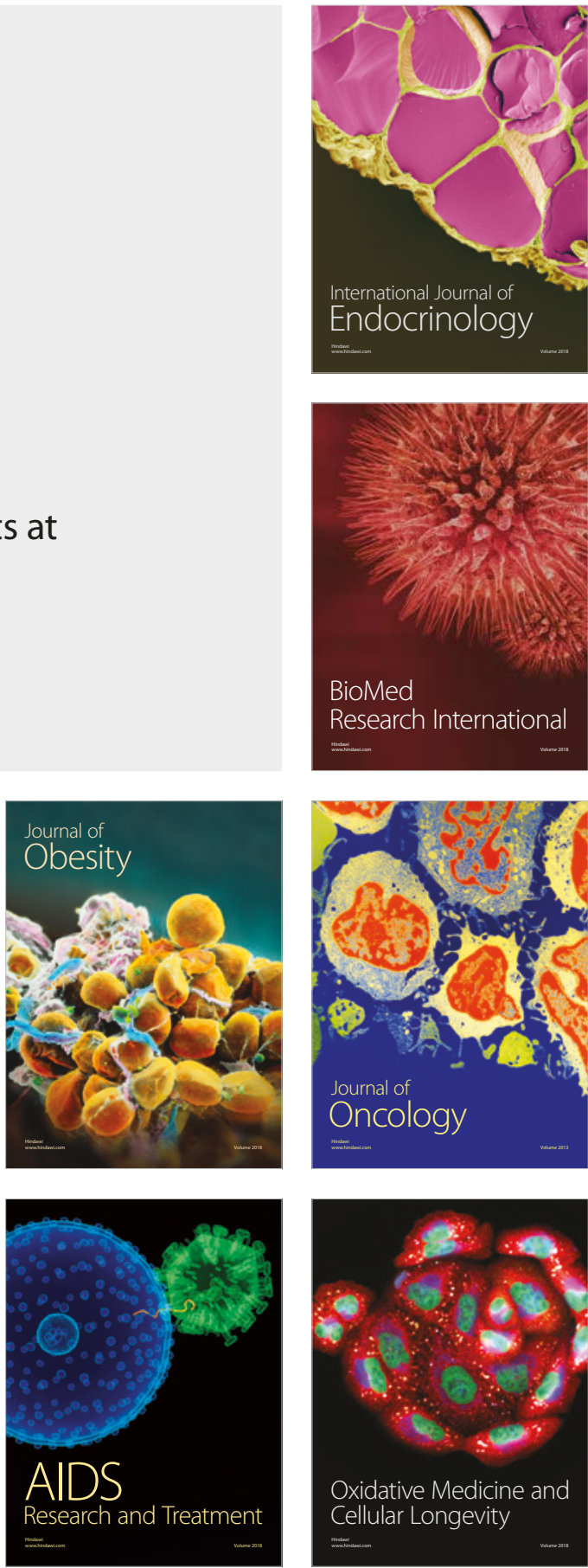